\title{
Weighted sum of per cell min-rate maximization for multi-cell downlink OFDMA with opportunistic DF relaying
}

\author{
Zhiwen Jin $^{1 *}$, Tao Wang ${ }^{2}$, Jibo Wei ${ }^{3}$ and Luc Vandendorpe ${ }^{1}$
}

\begin{abstract}
This paper considers a multi-cell relay-aided orthogonal frequency division multiple access (OFDMA) downlink system, in which all stations are coordinated by a central controller for resource allocation (RA). The decode-and-forward (DF) protocol with opportunistic relaying (OR) and high spectrum efficiency (HSE) is applied. The problem of maximizing the weighted sum of per cell min-rate (WSMR) with per-cell total power constraints is formulated, and its per-cell maximum fairness property is proven. An iterative RA algorithm is proposed to optimize mode selection (decision whether the relay should help or not), subcarrier assignment (MSSA) and power allocation (PA) alternatively. Each iteration is composed of the MSSA stage and the PA stage. During the MSSA stage, the original problem is decoupled into mixed integer linear programs (MILPS) with the tentative PA results, which can be solved by typical MILP solvers. To solve the MILPS more efficiently in polynomial time, a randomized rounding-based MSSA (RR-MSSA) algorithm and a direct rounding-based MSSA (DR-MSSA) algorithm are further proposed. During the PA stage, an algorithm based on single condensation and geometric programming (SCGP) PA is designed to optimize PA with the tentative MSSA results. The convergence and the per-cell user fairness of the proposed RA algorithm are proven. Finally, the performance of the RA algorithm and the benefits of using OR and the HSE protocol are illustrated through numerical experiments.
\end{abstract}

Keywords: OFDMA; Opportunistic DF relaying; Resource allocation; Per-cell user fairness; Cochannel interference mitigation

\section{Introduction}

In next-generation wireless communication networks, ubiquitous coverage and high data rate are strongly required. To achieve this goal, incorporating orthogonal frequency division multiple access (OFDMA) technology with emerging relaying technologies is highly investigated by both academia and industry. Specifically, OFDMA is able to combat the frequency-selective multi-path fading and flexible in applying dynamic radio resource allocation (RA) for performance improvement. On the other hand, the relaying technology has an attractive feature of coverage extension and data rate improvement [1].

\footnotetext{
*Correspondence: zhiwen.jin@student.uclouvain.be

1 ICTEAM institute (École Polytechnique de Louvain), Université catholique de Louvain, Louvain-la-Neuve 1348, Belgium

Full list of author information is available at the end of the article
}

For relay-aided OFDM(A) systems, the authors in [2] and [3] have proposed two efficient types of relaying, namely amplify and forward (AF) and decode and forward (DF). Recently, the DF relaying is receiving a lot of interest due to its simple processing at the relay. With DF relaying, symbols are transmitted in two time slots (TSs). During the first TS, the source broadcasts symbols on all subcarriers with the relay keeping quiet. During the second TS, except from the relay, the source might also broadcast symbols on subcarriers not used by the relay, as will be elaborated later. Adopting DF relaying, the authors in [4-23] have studied the RA problems for downlink OFDMA intensively.

In particular, the works in [4-7] have considered RA in OFDM systems with fixed relaying (FR) when the destination cannot or hardly hear from the source, meaning the source-to-destination (S-D) link is unavailable. Considering the case where the S-D link is available, the authors

\section{Springer}

(c) 2014 Jin et al: licensee Springer. This is an Open Access article distributed under the terms of the Creative Commons

Attribution License (http://creativecommons.org/licenses/by/2.0), which permits unrestricted use, distribution, and reproduction in any medium, provided the original work is properly credited. 
in [8-23] have studied RA in systems with opportunistic relaying $(\mathrm{OR})$, sometimes termed as selection relaying. To start with, a low spectrum efficiency (LSE) protocol was studied in [8-13], when only the relay broadcasts symbols during the second TS. Specifically, each transmission is carried out in two TSs using either the relay-aided mode or the direct mode. As for a relay-aided transmission, during the first TS, a symbol is first broadcast by the base station (BS) at a subcarrier $k$. Both the relay station (RS) and the targeted mobile station (MS) receive this symbol. Then, during the second TS, the RS decodes the received symbol and retransmits it to the targeted MS at a subcarrier $l$. Note that subcarrier $l$ might not be the same as subcarrier $k$, which means subcarrier pairing (SP) can be implemented [17,24]. Finally, the MS chooses to decode the source symbol from either the received symbols during both TSs using some combination methods or the received symbol during the second TS only. For a direct mode transmission, the MS only uses what is received from the BS. Note that adopting this LSE protocol, the BS keeps quiet with unpaired subcarriers unused during the second TS, which causes a waste of the limited spectrum resource. To address this issue, the authors in [14-22] have proposed and studied improved high spectrum efficiency (HSE) protocols, which allow new symbols to be transmitted on the unpaired subcarriers during the second TS. In this paper, the HSE protocol will be considered.

Note that all these papers model the cochannel interference (CCI) as additive background noise and only consider RA in single-cell situations. This is reasonable only when the frequency reuse factor $1 / W$ is low, where $W$ denotes the number of cell clusters that cannot use the same frequencies for transmission. However, in nextgeneration cellular systems, aggressive frequency reuse is recommended due to its ability to achieve higher system capacity [25]. Here, the CCI becomes a key factor affecting the system performance and thus cannot be ignored [26].

Considering the CCI in multi-cell DF relay-aided OFDMA systems, RA algorithms have been proposed in [27] and [28] when powers are uniformly allocated to all stations. Considering subcarrier allocation and power allocation jointly, the papers [26] and [29] have recently formulated and solved the sum rate maximized RA problem in multi-cell OFDMA downlink systems aided by DF RSs in each cell. However, fairness was not considered there. Recently in [30], the maximization of the weighted sum of per cell min-rate (WSMR) was considered for multi-cell OFDMA systems without relaying. The corresponding optimum RA leads to per-cell maximum fairness and different priorities in different cells. The authors only illustrate the per-cell fairness by experiments without mathematical proof.
Compared with the above existing works, the contributions of the current paper are listed below:

- We formulate the WSMR maximized joint RA problem in multi-cell OFDMA downlink systems aided by a RS in each cell. To start with, the DF protocol with OR and HSE is considered without signal combining. It will be shown that the system performance can be enhanced largely by using OR and the HSE protocol. Note that when modeling the inter-cell CCI of a subcarrier in a selected cell, instead of using an additional integer variable to indicate whether a node in an interfering cell transmits data on this subcarrier or not, we use the corresponding power value to do it. This choice is motivated to simplify the system sum rate expression and facilitate the algorithm design.

- We unveil and prove the per-cell maximum fairness feature of the formulated RA problem. Specifically, with any feasible mode selection and subcarrier assignment (MSSA) result, the optimum power allocation results in the same transmission rates for all users in the same cell.

- We propose an iterative RA algorithm to optimize the MSSA and the power allocation (PA) alternatively with the WSMR keeping increasing. Each iteration is composed of the MSSA stage and the PA stage. During the MSSA stage, the original problem is decoupled into mixed integer linear programs (MILPs), which can be solved by typical MILP solvers. To solve the MILPs more efficiently in polynomial time, we further propose the randomized rounding-based MSSA (RR-MSSA) algorithm and the direct rounding-based MSSA (DR-MSSA) algorithm at this stage. During the PA stage, an algorithm based on single condensation and geometric programming PA (SCGP) is used to optimize PA with the tentative MSSA results. The convergence and the per-cell user fairness of the proposed RA algorithm are proven.

The rest of this paper is organized as follows: First, the considered system model is presented in the next section. Then, the RA problem is formulated and its fairness property is discussed in Section 3. After that, the proposed algorithms are described in Section 4. Furthermore, the effectiveness and convergence of the proposed RA algorithms as well as the benefits of using OR and the HSE protocol are illustrated by numerical experiments in Section 5. Finally, some conclusions are drawn in Section 6.

\section{System description}

Let us consider a cellular downlink OFDMA system with $N$ cells coordinated by a central controller for RA. In each cell, the data is transmitted from a source to $U$ 
destinations with the help of a DF relay. For each link, the frequency-selective channel is transformed into $K$ parallel subchannels by using OFDM with sufficiently long cyclic prefix. The data transmission is carried out in two TSs choosing either relay-aided mode or direct mode. During the first TS, a symbol is first broadcast by the BS at a subcarrier and received by the RS and the MS. In relay-aided mode, the RS decodes the received symbol and relays it to the targeted MS over this subcarrier with the BS keeping quiet at the subcarrier during the second TS. The destination only decodes the symbol received during the second TS. If direct transmission is used, the targeted destination decodes the symbol received during the first TS. Also, another symbol is broadcast by the source at this subcarrier during the second TS, which is received and decoded by this destination.

With OFDMA, each subcarrier can be allocated to only one destination in each cell. In this paper, we first assume that the coherence time of each link is sufficiently long for implementing the RA in the central controller, which has perfect knowledge of the system channel state information. Moreover, we assume that the optimized RA can be correctly distributed to all nodes. Lastly, perfect timing and carrier synchronization is assumed. Note that an upper bound on the system performances is obtained by assuming the above idealities.

\subsection{Transmission procedure}

Let us describe the data transmission procedure inside one selected cell $n$, which is impaired by cochannel interference from the other cells. The transmission procedures in other cells are the same. Specifically in cell $n$, symbols are transmitted in either relay-aided mode or direct mode, as will be elaborated in the following.

We first consider the relay-aided data transmission. The source $s_{n}$ first produces a symbol $\sqrt{P_{\mathrm{s}_{n}, t_{1}}^{k}} x_{\mathrm{s}_{n}, t_{1}}^{k}$ at subcarrier $k$ during the first TS, while the transmitter of the relay $r_{n}$ remains idle. Here, $x_{\mathrm{s}_{n}, t_{1}}^{k}$ denotes the normalized symbol (meaning $E\left\{\left|x_{s_{n}, t_{1}}^{k}\right|^{2}\right\}=1$ ) transmitted by $s_{n}$ at subcarrier $k$ during the first TS, and $P_{\mathrm{s}_{n}, t_{1}}^{k}$ denotes the corresponding transmit power. Simultaneously in an interfering cell $n^{\prime}$, a symbol $\sqrt{P_{\mathrm{s}_{n^{\prime}}, t_{1}}^{k}} x_{\mathrm{s}_{n^{\prime}}, t_{1}}^{k}$ is also produced from the interfering source $s_{n^{\prime}}$ at the same subcarrier. Note that instead of using an additional integer variable to indicate whether $\mathrm{s}_{n^{\prime}}$ transmits data on subcarrier $k$ or not, we use $\sqrt{P_{s_{n^{\prime}}, t_{1}}^{k}}$ to do so. Specifically, $P_{\mathrm{s}_{n^{\prime}}, t_{1}}^{k}>0$ means that $\mathrm{s}_{n^{\prime}}$ uses subcarrier $k$ for data transmission during the first TS, and $P_{\mathrm{s}_{n^{\prime}}, t_{1}}^{k}=0$ means that $s_{n^{\prime}}$ does not transmit at the subcarrier $k$ during the first TS. This choice is motivated to simplify the system sum rate expression and facilitate the algorithm design. At the end of the first TS, the signal received by $\mathrm{r}_{n}$ for subcarrier $k$ can be expressed as

$$
\begin{aligned}
y_{\mathrm{r}_{n}}^{k}= & \sqrt{P_{\mathrm{s}_{n}, t_{1}}^{k}} h_{\mathrm{s}_{n}, \mathrm{r}_{n}}^{k} x_{\mathrm{s}_{n}, t_{1}}^{k}+v_{\mathrm{r}_{n}}^{k} \\
& +\sum_{n^{\prime}=1, n^{\prime} \neq n}^{k} \sqrt{P_{\mathrm{s}_{n^{\prime}}, t_{1}}^{k}} h_{\mathrm{s}_{n^{\prime}}, \mathrm{r}_{n}}^{k} x_{\mathrm{s}_{n^{\prime}}, t_{1}}^{k}
\end{aligned}
$$

where $v_{\mathrm{r}_{n}}^{k}$ denotes the additive white Gaussian noise (AWGN) at subcarrier $k$ and $\mathrm{r}_{n}$ during the first TS. $h_{\mathrm{s}_{n^{\prime}}, \mathrm{r}_{n}}^{k}$ denotes the channel frequency response (CFR) for subcarrier $k$ from $\mathrm{s}_{n^{\prime}}$ to $\mathrm{r}_{n}$.

During the second TS, the relay $\mathrm{r}_{n}$ reencodes the decoded symbol and forwards $\sqrt{P_{\mathrm{r}_{n}}^{k}} x_{\mathrm{r}_{n}}^{k}$ at a subcarrier $k$. Here, $x_{\mathrm{r}_{n}}^{k}=x_{\mathrm{s}_{n}, t_{1}}^{k}$. The source $\mathrm{s}_{n}$ transmits nothing on this subcarrier, meaning that $P_{\mathrm{s}_{n}, t_{2}}^{k}=0$. Here, $P_{\mathrm{r}_{n}}^{k}$ and $P_{\mathrm{s}_{n}, t_{2}}^{k}$ denote the transmit power allocated to $r_{n}$ and $\mathrm{s}_{n}$, respectively, at subcarrier $k$ during the second TS. At the same time, in an interfering cell $n^{\prime}, \mathrm{r}_{n^{\prime}}$ and $\mathrm{s}_{n^{\prime}}$ also transmit $\sqrt{P_{\mathrm{r}_{n^{\prime}}}^{k}} x_{\mathrm{r}_{n^{\prime}}}^{k}$ and $\sqrt{P_{\mathrm{s}_{n^{\prime}}, t_{2}}^{k}} x_{\mathrm{s}_{n^{\prime}}, t_{2}}^{k}$ at subcarrier $k$. Remembering that at most only one power value out of $P_{\mathrm{r}_{n^{\prime}}}^{k}$ and $P_{\mathrm{s}_{n^{\prime}}, t_{2}}^{k}$ can be non-zero. More specifically, when subcarrier $k$ of cell $n^{\prime}$ is assigned, the relay mode for data transmission $P_{\mathrm{s}_{n^{\prime}}, t_{2}}^{k}=0$. Otherwise, $P_{\mathrm{r}_{n^{\prime}}}^{k}=0$. Here again $E\left\{\left|x_{\mathrm{r}_{n^{\prime}}}^{k}\right|^{2}\right\}=1$ and $E\left\{\left|x_{\mathrm{S}_{n^{\prime}}, t_{2}}^{k}\right|^{2}\right\}=1$. At the end of the second TS, the signal received by the targeted destination $\mathrm{d}_{u n}$ at subcarrier $k$ can be expressed as

$$
\begin{aligned}
y_{\mathrm{d}_{u n}, t_{2}}^{k}= & \sqrt{P_{\mathrm{r}_{n}}^{k}} h_{\mathrm{r}_{n}, \mathrm{~d}_{u n}}^{k} x_{\mathrm{r}_{n}}^{k}+v_{\mathrm{d}_{u n}, t_{2}}^{k} \\
& +\sum_{n^{\prime}=1, n^{\prime} \neq n}^{N} \sqrt{P_{\mathrm{s}_{n^{\prime}}, t_{2}}^{k}} h_{\mathrm{s}_{n^{\prime}}, \mathrm{d}_{u n}}^{k} x_{\mathrm{s}_{n^{\prime}}, t_{2}}^{k} \\
& +\sum_{n^{\prime}=1, n^{\prime} \neq n}^{N} \sqrt{P_{\mathrm{r}_{n^{\prime}}}^{k}} h_{\mathrm{r}_{n^{\prime}}, \mathrm{d}_{u n}}^{k} x_{\mathrm{r}_{n^{\prime}}}^{k}
\end{aligned}
$$

where $v_{\mathrm{d}_{u n}, t_{2}}^{k}$ denotes the AWGN corrupting $\mathrm{d}_{u n}$ at subcarrier $k$ during the second TS, $h_{\mathrm{r}_{n^{\prime}}, \mathrm{d}_{u n}}^{k}$ denotes the CFR for subcarrier $k$ from $\mathrm{r}_{n^{\prime}}$ to $\mathrm{d}_{u n}$, and $h_{\mathrm{s}_{n^{\prime}}, \mathrm{d}_{u n}}^{k}$ denotes the CFR of subcarrier $k$ from $s_{n^{\prime}}$ to $d_{u n}$.

Let us now describe the direct data transmission in cell $n$. During the first TS, $\mathrm{s}_{n}$ broadcasts $\sqrt{P_{\mathrm{s}_{n}, t_{1}}^{k}} x_{\mathrm{s}_{n}, t_{1}}^{k}$ at subcarrier $k$. The targeted destination $\mathrm{d}_{u n}$ receives signals from all sources. The signal received by $\mathrm{d}_{u n}$ at subcarrier $k$ can be expressed as

$$
\begin{aligned}
z_{\mathrm{d}_{u n}, t_{1}}^{k}= & \sqrt{P_{\mathrm{s}_{n}, t_{1}}^{k}} h_{\mathrm{s}_{n}, \mathrm{~d}_{u n}}^{k} x_{\mathrm{s}_{n}, t_{1}}^{k}+v_{\mathrm{d}_{u n}, t_{1}}^{k} \\
& +\sum_{n^{\prime}=1, n^{\prime} \neq n}^{N} \sqrt{P_{\mathrm{s}_{n^{\prime}}, t_{1}}^{k}} h_{\mathrm{s}_{n^{\prime}}, \mathrm{d}_{u n}}^{k} x_{\mathrm{s}_{n^{\prime}}, t_{1}}^{k}
\end{aligned}
$$


where $v_{\mathrm{d}_{u n}, t_{1}}^{k}$ denotes the AWGN corrupting $\mathrm{d}_{u n}$ at subcarrier $k$ during the first TS.

During the second TS, another symbol $\sqrt{P_{\mathrm{s}_{n}, t_{2}}^{k}} x_{\mathrm{s}_{n}, t_{2}}^{k}$ is broadcast by $\mathrm{s}_{n}$ at subcarrier $k$ and received by the destination $\mathrm{d}_{u n}$. The received signal can be expressed as

$$
\begin{aligned}
z_{\mathrm{d}_{u n}, t_{2}}^{k}= & \sqrt{P_{\mathrm{s}_{n}, t_{2}}^{k}} h_{\mathrm{s}_{n}, \mathrm{~d}_{u n}}^{k} x_{\mathrm{s}_{n}, t_{2}}^{k}+v_{\mathrm{d}_{u n}, t_{2}}^{k} \\
& +\sum_{n^{\prime}=1, n^{\prime} \neq n}^{N} \sqrt{P_{\mathrm{s}_{n^{\prime}}, t_{2}}^{k}} h_{\mathrm{s}_{n^{\prime}},}^{k} \mathrm{~d}_{u n} x_{\mathrm{s}_{n^{\prime}}, t_{2}}^{k} \\
& +\sum_{n^{\prime}=1, n^{\prime} \neq n}^{N} \sqrt{P_{\mathrm{r}_{n^{\prime}}}^{k}} h_{\mathrm{r}_{n^{\prime}}, \mathrm{d}_{u n}}^{k} x_{\mathrm{r}_{n^{\prime}}}^{k} .
\end{aligned}
$$

\subsection{Weighted sum of per cell minimum rate}

In order to formulate the WSMR, we now introduce binary variables $a_{u n}^{k}$ and $b_{u n}^{k}$ to describe the mode selection and subcarrier assignment in both TSs. To be more specific, $a_{u n}^{k}=1$ indicates that subcarrier $k$ is allocated for data transmission to $\mathrm{d}_{u n}$ in direct mode during the first TS, and so is subcarrier $k$ to $\mathrm{d}_{u n}$ during the second TS. $b_{u n}^{k}=1$ indicates that subcarrier $k$ is allocated for data transmission to $\mathrm{d}_{u n}$ aided by $\mathrm{r}_{n}$.

We assume $\left\{v_{\mathrm{r}_{n}}^{k}, v_{\mathrm{d}_{u n}, t_{2}}^{k}, v_{\mathrm{d}_{u n}, t_{1}}^{k}\right\}$ are independent zeromean circular Gaussian random variables with the same variance $\sigma^{2}$. After some mathematical calculations, the sum rate achievable for the direct mode subcarrier $k$ is given by

$$
R_{u n, 1}^{k}=\ln \left(1+\Upsilon_{\mathrm{d}_{u n}, t_{1}}^{k}\right)+\ln \left(1+\Upsilon_{\mathrm{d}_{u n}, t_{2}}^{k}\right)
$$

in nats/two-TSs, where

$$
\Upsilon_{\mathrm{d}_{u n}, t_{1}}^{k}=\frac{P_{\mathrm{s}_{n}, t_{1}}^{k} G_{\mathrm{s}_{n}, \mathrm{~d}_{u n}}^{k}}{f_{\mathrm{d}_{u n}, t_{1}}^{k}}
$$

denotes the SINR associated with the decoding of $x_{\mathrm{s}_{n}, t_{1}}^{k}$ from $y_{\mathrm{d}_{u n}, t_{1}}^{k}$ at $\mathrm{d}_{u n}$ during the first TS. $f_{\mathrm{d}_{u n}, t_{1}}^{k}=\sigma^{2}+$ $\sum_{n^{\prime}=1, n^{\prime} \neq n}^{N} P_{\mathrm{s}_{n^{\prime}}, t_{1}}^{k} G_{\mathrm{s}_{n^{\prime}}, \mathrm{d}_{u n}}^{k}$ denotes the sum power of the AWGN and the interference received by $\mathrm{d}_{u n}$ at subcarrier $k$ during the first TS. $G_{\mathrm{s}_{n^{\prime}}, \mathrm{d}_{u n}}^{k}=\left|h_{\mathrm{s}_{n^{\prime}}, \mathrm{d}_{u n}}^{k}\right|^{2}$ denotes the channel gain of subcarrier $k$ from $s_{n^{\prime}}$ to $d_{u n}$.

$$
\Upsilon_{\mathrm{d}_{u n}, t_{2}}^{k}=\frac{P_{\mathrm{s}_{n}, t_{2}}^{k} G_{\mathrm{s}_{n}, \mathrm{~d}_{u n}}^{k}}{f_{\mathrm{d}_{u n}, t_{2}}^{k}}
$$

denotes the SINR associated with the decoding of $x_{\mathrm{s}_{n}, t_{2}}^{k}$ from $y_{\mathrm{d}_{u n}, t_{2}}^{k}$ at $\mathrm{d}_{u n}$ during the second TS. $f_{\mathrm{d}_{u n}, t_{2}}^{k}=\sigma^{2}+$ $\sum_{n^{\prime}=1, n^{\prime} \neq n}^{N} P_{\mathrm{s}_{n^{\prime}}, t_{2}}^{k} G_{\mathrm{s}_{n^{\prime}}, \mathrm{d}_{u n}}^{k}+\sum_{n^{\prime}=1, n^{\prime} \neq n}^{N} P_{\mathrm{r}_{n^{\prime}}}^{k} G_{\mathrm{r}_{n^{\prime}}, \mathrm{d}_{u n}}^{k}$ denotes the sum power of the AWGN and the interference received by $\mathrm{d}_{u n}$ at subcarrier $k$ during the second TS. $G_{\mathrm{r}_{n}, \mathrm{~d}_{u n}}^{k}=$ $\left|h_{\mathrm{r}_{n}, \mathrm{~d}_{u n}}^{k}\right|^{2}$ denotes the channel gain of subcarrier $k$ from $\mathrm{r}_{n}$ to $\mathrm{d}_{u n}$.

Also, the maximum achievable rate for subcarrier $k$ when allocated to $d_{u n}$ in relay-aided mode is given by [3]

$$
R_{u n, 2}^{k}=\min \left(\ln \left(1+\Gamma_{\mathrm{r}_{n}}^{k}\right), \ln \left(1+\Gamma_{\mathrm{d}_{u n}, t_{2}}^{k}\right)\right)
$$

in nats/two-TSs, where

$$
\Gamma_{\mathrm{r}_{n}}^{k}=\frac{P_{\mathrm{s}_{n}, t_{1}}^{k} G_{\mathrm{s}_{n}, \mathrm{r}_{n}}^{k}}{f_{\mathrm{r}_{n}}^{k}}
$$

denotes the signal-to-interference-plus-noise ratio (SINR) associated with decoding $x_{\mathrm{s}_{n}, t_{1}}^{k}$ from $y_{\mathrm{r}_{n}}^{k}$ at $\mathrm{r}_{n}$ during the first TS, $f_{\mathrm{r}_{n}}^{k}=\sigma^{2}+\sum_{n^{\prime}, n^{\prime} \neq n} P_{\mathrm{s}_{n^{\prime}}, t_{1}}^{k} G_{\mathrm{s}_{n^{\prime}}, \mathbf{r}_{n}}^{k}$ denotes the sum power of the AWGN and the interference received by $\mathrm{r}_{n}$ at subcarrier $k$ during the first TS, and $G_{\mathrm{s}_{n}, \mathrm{r}_{n}}^{k}=$ $\left|h_{\mathrm{s}_{n}, \mathrm{r}_{n}}^{k}\right|^{2}$ denotes the channel gain of subcarrier $k$ from $\mathrm{s}_{n}$ to $r_{n}$.

$$
\Gamma_{\mathrm{d}_{u n}, t_{2}}^{k}=\frac{P_{\mathrm{r}_{n}}^{k} G_{\mathrm{r}_{n}, \mathrm{~d}_{u n}}^{k}}{f_{\mathrm{d}_{u n}, t_{2}}^{k}}
$$

denotes the SINR associated with decoding $x_{\mathbf{r}_{n}}^{k}$, which equals $x_{\mathrm{s}_{n}, t_{1}}^{k}$, from $y_{\mathrm{d}_{u n}, \mathrm{r}_{n}}^{k}$ at $\mathrm{d}_{u n}$ during the second TS.

Thus, the WSMR of the considered system is denoted as

$$
\begin{aligned}
R(\mathbf{P}, \mathbf{A}, \mathbf{B})=\sum_{n=1}^{N} \omega_{n} \min _{u \in \mathcal{U}_{n}}( & \sum_{k=1}^{K} a_{u n}^{k} R_{u n, 1}^{k}\left(\mathbf{P}^{k}\right) \\
& \left.+\sum_{k=1}^{K} b_{u n}^{k} R_{u n, 2}^{k}\left(\mathbf{P}^{k}\right)\right),
\end{aligned}
$$

where $\mathbf{P}^{k}=\left[\left[\mathbf{P}_{\mathbf{1}}^{\mathbf{k}}\right]^{\mathrm{T}}, \ldots,\left[\mathbf{P}_{\mathbf{N}}^{\mathbf{k}}\right]^{\mathrm{T}}\right]^{\mathrm{T}}, \mathbf{P}_{\mathbf{n}}^{\mathbf{k}}=\left[P_{\mathrm{s}_{n}, t_{1}}^{k}, P_{\mathrm{s}_{n}, t_{2}}^{k}\right.$, $\left.P_{\mathbf{r}_{n}}^{k}\right]^{\mathrm{T}}, \mathbf{A}=\left\{a_{u n}^{k}\right\}$, and $\mathbf{B}=\left\{b_{u n}^{k}\right\} \cdot \omega_{n}$ denotes the weight of the minimal rate in cell $n$, and $\mathcal{U}_{n}$ denotes the MS set in cell $n$.

\section{Problem formulation and its property}

\subsection{Problem formulation}

We consider maximizing the WSMR under per-cell total power constraints. The optimization variables are the transmission mode for each subcarrier, the subcarrier assignments, and the power allocations at the sources and 
the relays. According to the system model, the considered RA problem can be formulated as

$$
\begin{aligned}
& \max _{\mathbf{P}, \mathbf{A}, \mathbf{B}} R(\mathbf{P}, \mathbf{A}, \mathbf{B}) \\
& \text { s.t. } \\
& C 1:\left(\sum_{u=1}^{U} a_{u n}^{k}+\sum_{u=1}^{U} b_{u n}^{k}\right) \leq 1, \forall n, k, \\
& C 2: a_{u n}^{k} \in\{0,1\}, b_{u n}^{k} \in\{0,1\}, \forall u, n, k, \\
& C 3: \sum_{k=1}^{K} P_{\mathrm{s}_{n}, t_{1}}^{k}+\sum_{k=1}^{K} P_{\mathrm{s}_{n}, t_{2}}^{k}+\sum_{k=1}^{K} P_{\mathrm{r}_{n}}^{k} \leq P_{t, n}, \forall n, \\
& C 4: P_{\mathrm{s}_{n}, t_{1}}^{k} \geq 0, P_{\mathrm{s}_{n}, t_{2}}^{k} \geq 0, P_{\mathrm{r}_{n}}^{k} \geq 0, \forall n, k, \\
& C 5: P_{\mathrm{s}_{n}, t_{1}}^{k}\left(1-\sum_{u=1}^{U} a_{u n}^{k}-\sum_{u=1}^{U} b_{u n}^{k}\right)=0, \forall n, k, \\
& C 6: P_{\mathrm{s}_{n}, t_{2}}^{k}\left(1-\sum_{u=1}^{U} a_{u n}^{k}\right)=0, \forall n, k, \\
& C 7: P_{\mathrm{r}_{n}}^{k}\left(1-\sum_{u=1}^{U} b_{u n}^{k}\right)=0, \forall n, k .
\end{aligned}
$$

Here, $C 1$ and $C 2$ ensure that each subcarrier $k$ during the first TS can select only one mode (direct/relay-aided) to transmit data towards only one destination $\mathrm{d}_{u n}$. Moreover, $C 3$ and $C 4$ ensure that the consumed sum power for each cell is less than its available sum power. This type of power constraints give an upper bound of the system performance. In practice, each node (source, relay) in each cell will have an individual power constraint. Finally, C5, $C 6$, and $C 7$ guarantee that no data is transmitted on an unused subcarrier, and subcarrier $k$ is used by only one node (either the source or the relay) in each cell during the second TS.

\subsection{Fairness property}

To investigate the fairness property of the formulated problem, let us denote by $R_{u n}$ the sum rate of each user $u$ in each cell $n, \mathcal{U}_{n}$ the set of users in cell $n, \tilde{\mathcal{U}}_{n} \subset \mathcal{U}_{n}$ the set of users with the minimum rate in cell $n, \tilde{\mathcal{V}}_{n}$ the set of other users (the complimentary set of $\left.\tilde{\mathcal{U}}_{n}\right)$, and $\mathcal{S}_{\tilde{u} n}\left(\mathcal{S}_{\tilde{v} n}\right)$ the set of subcarriers allocated to MS $d_{\tilde{u} n}\left(d_{\tilde{v} n}\right)$, where $\tilde{u} \in \tilde{\mathcal{U}}_{n}\left(\tilde{v} \in \tilde{\mathcal{V}}_{n}\right)$. Note that $\tilde{\mathcal{V}}_{n} \neq \emptyset$ means that

$$
\min _{\tilde{v} \in \tilde{\mathcal{V}}_{n}} R_{\tilde{v} n}>\min _{\tilde{u} \in \tilde{\mathcal{U}}_{n}} R_{\tilde{u} n}=\min _{u \in \mathcal{U}_{n}} R_{u n} .
$$

Then, for each subcarrier $k$, there exists three states considering which one of the two user sets does the allocated user belong to in each cell. Specifically, state 1 corresponds to $k \in \mathcal{S}_{\tilde{u} n}, \forall n$, meaning that in each cell, subcarrier $k$ is allocated to a user with the minimum rate of that cell. State 2 corresponds to $k \in \mathcal{S}_{\tilde{v} n}, \forall n$, meaning that in each cell, subcarrier $k$ is allocated to a user with a rate larger than the minimum rate of that cell. State 3 corresponds to $\exists n_{1}, n_{2}$ such that $k \in \mathcal{S}_{\tilde{u} n_{1}}$ and $k \in \mathcal{S}_{\tilde{v} n_{2}}$, meaning other possible allocation results for subcarrier $k$ in all cells. Note that when all subcarriers are in state 1, per-cell user fairness is reached, meaning all users inside the same cell obtain the same rate.

We first consider the following lemma, which will be used later in this section.

Lemma 1. Given any feasible values of variables $\mathbf{A}, \mathbf{B}$ and $\mathbf{P}$, if there exists $n$ and $k$ where $\tilde{\mathcal{V}}_{n} \neq \emptyset$ and $k \in \mathcal{S}_{\tilde{v} n}$ (meaning that there exists subcarrier $k$ in state 2 or state 3$)$, then part of the feasible powers at this subcarrier $k$ in each cell $\left(\mathbf{P}_{\mathbf{n}}^{\mathbf{k}}=\left\{P_{\mathrm{s}_{n}, t_{1}}^{k}, P_{\mathrm{s}_{n}, t_{2}}^{k}, P_{\mathrm{r}_{n}}^{k}\right\}\right.$ and $\left.\mathbf{P}_{n^{\prime}}^{k}, \forall n^{\prime} \neq n\right)$ can be saved while keeping the WSMR of the system non-decreased.

Proof. Considering the subcarrier $k$ in cell $n$ where $\tilde{\mathcal{V}}_{n} \neq$ $\emptyset$ and $k \in \mathcal{S}_{\tilde{v} n}$, we now introduce two sets of cells $\mathcal{N}_{1}^{k}=$ $\left\{n \mid k \in \mathcal{S}_{\tilde{u} n}\right\}$ and $\mathcal{N}_{2}^{k}=\left\{n \mid \tilde{\mathcal{V}}_{n} \neq \emptyset, k \in \mathcal{S}_{\tilde{v} n}\right\}$. Note that when $k$ is in state $2, \mathcal{N}_{1}^{k}=\emptyset$ and $\mathcal{N}_{2}^{k} \neq \emptyset$. Also, when $k$ is in state $3, \mathcal{N}_{1}^{k} \neq \emptyset$ and $\mathcal{N}_{2}^{k} \neq \emptyset$.

Let us consider the case when $k$ is in state 3 . We define

$$
\tilde{\mathbf{P}}_{n}^{k}\left(s^{k}\right)= \begin{cases}s^{k} * \mathbf{P}_{\mathbf{n}}^{\mathbf{k}} & n \in \mathcal{N}_{2}^{k} \\ \mathbf{P}_{\mathbf{n}}^{\mathbf{k}} & n \in \mathcal{N}_{1}^{k}\end{cases}
$$

where $s^{k} \in(0,1], \tilde{R}_{u n}^{k}\left(\tilde{\mathbf{P}}^{k}\left(s^{k}\right)\right)=a_{u n}^{k} R_{u n, 1}^{k}\left(\tilde{\mathbf{P}}^{k}\left(s^{k}\right)\right)$ $+b_{u n}^{k} R_{u n, 2}^{k}\left(\tilde{\mathbf{P}}^{k}\left(s^{k}\right)\right)$, and $\tilde{\mathbf{P}}^{k}\left(s^{k}\right)=\left[\left[\tilde{\mathbf{P}}_{1}^{k}\left(s^{k}\right)\right]^{\mathrm{T}}, \ldots\right.$, $\left.\left[\tilde{\mathbf{P}}_{N}^{k}\left(s^{k}\right)\right]^{\mathrm{T}}\right]^{\mathrm{T}}$. Please note that $s^{k}$ is the same for all cells. $\forall n \in \mathcal{N}_{2}^{k}, \Upsilon_{\mathrm{d}_{\tilde{v}}, t_{1}}^{k}$ can be denoted as

$$
\frac{s^{k} P_{\mathrm{s}_{n}, t_{1}}^{k} G_{\mathrm{s}_{n},}^{k} \mathrm{~d}_{\tilde{\nu} n}}{\sigma^{2}+\sum_{n^{\prime} \in \mathcal{N}_{1}^{k}} P_{\mathrm{s}_{n^{\prime}}, t_{1}}^{k} G_{\mathrm{s}_{n^{\prime}}, \mathrm{d}_{\tilde{\nu} n}}^{k}+\sum_{n^{\prime} \neq n, n^{\prime} \in \mathcal{N}_{2}^{k}} s^{k} P_{\mathrm{s}_{n^{\prime}}, t_{1}}^{k} G_{\mathrm{s}_{n^{\prime}}, \mathrm{d}_{\tilde{v} n}}^{k}},
$$

which can be further written as

$$
\frac{P_{\mathrm{s}_{n}, t_{1}}^{k} G_{\mathrm{s}_{n}, \mathrm{~d}_{\tilde{\nu} n}}^{k}}{\frac{\sigma^{2}}{s^{k}}+\sum_{n^{\prime} \in \mathcal{N}_{1}^{k}} \frac{P_{\mathrm{s}_{n^{\prime}}, t_{1}}^{k} G_{\mathrm{s}_{n^{\prime}}, \mathrm{d}_{\tilde{\nu} n}}^{k}}{s^{k}}+\sum_{n^{\prime} \neq n, n^{\prime} \in \mathcal{N}_{2}^{k}} P_{\mathrm{s}_{n^{\prime}}, t_{1}}^{k} G_{\mathrm{s}_{n^{\prime}}, \mathrm{d}_{\tilde{\nu} n}}^{k}} .
$$

It is obvious that $\Upsilon_{\mathrm{d}_{\tilde{\nu} n}, t_{1}}^{k}$ is an increasing function of $s^{k}$. Similarly, $\Upsilon_{\mathrm{d}_{\tilde{\nu}}, t_{2}}^{k}, \Gamma_{\mathrm{r}_{n}}^{k}$, and $\Gamma_{\mathrm{d}_{\tilde{\nu}}, t_{2}}^{k}$ are also increasing functions of $s^{k}$. Thus, $\tilde{R}_{\tilde{v} n}^{k}\left(\tilde{\mathbf{P}}^{k}\left(s^{k}\right)\right)$ is an increasing function of $s^{k}$. 
$\forall n \in \mathcal{N}_{1}^{k}, \Upsilon_{\mathrm{d}_{\tilde{u} n}, t_{1}}^{k}$ can be denoted as

$$
\frac{P_{\mathrm{s}_{n}, t_{1}}^{k} G_{\mathrm{s}_{n}, \mathrm{~d}_{\tilde{u} n}}^{k}}{\sigma^{2}+\sum_{n^{\prime} \neq n, n^{\prime} \in \mathcal{N}_{1}^{k}} P_{\mathrm{s}_{n^{\prime}}, t_{1}}^{k} G_{\mathrm{s}_{n^{\prime}}, \mathrm{d}_{\tilde{u} n}}^{k}+\sum_{n^{\prime} \in \mathcal{N}_{2}^{k}} s^{k} P_{\mathrm{s}_{n^{\prime}}, t_{1}}^{k} G_{\mathrm{s}_{n^{\prime}}, \mathrm{d}_{\tilde{u} n}}^{k}} .
$$

It is obvious that $\Upsilon_{\mathrm{d}_{\tilde{u} n}, t_{1}}^{k}$ is a decreasing function of $s^{k}$. Similarly, $\Upsilon_{\mathrm{d}_{\tilde{u} n}, t_{2}}^{k}, \Gamma_{\mathrm{r}_{n}}^{k}$, and $\Gamma_{\mathrm{d}_{\tilde{u} n}, t_{2}}^{k}$ are also decreasing functions of $s^{k}$. Thus, $\tilde{R}_{\tilde{u} n}^{k}\left(\tilde{\mathbf{P}}^{k}\left(s^{k}\right)\right)$ is a decreasing function of $s^{k}$.

Note that $\tilde{\mathbf{P}}^{k}\left(s^{k}\right) \leq \tilde{\mathbf{P}}^{k}(1)=\mathbf{P}^{k}$, where the inequality should be understood as elementwise. Thus, $\exists s_{0}^{k} \in(0,1), \epsilon_{n}^{k}>0, \eta_{n^{\prime}}^{k}>0$, such that $\forall n \in$ $\mathcal{N}_{2}^{k}, \tilde{R}_{\tilde{v} n}^{k}\left(\tilde{\mathbf{P}}^{k}\left(s_{0}^{k}\right)\right)=\tilde{R}_{\tilde{v} n}^{k}\left(\tilde{\mathbf{P}}^{k}(1)\right)-\epsilon_{n}^{k}=R_{\tilde{v} n}^{k}\left(\mathbf{P}^{k}\right)-$ $\epsilon_{n}^{k}, \tilde{R}_{\tilde{v} n}=R_{\tilde{v} n}-\epsilon_{n}^{k}>\min _{u \in \mathcal{U}_{n}} R_{u n}$ and $\forall n^{\prime} \in$ $\mathcal{N}_{1}^{k}, \tilde{R}_{\tilde{u} n^{\prime}}^{k}\left(\tilde{\mathbf{P}}^{k}\left(s_{0}^{k}\right)\right)=R_{\tilde{u} n^{\prime}}^{k}\left(\tilde{\mathbf{P}}^{k}(1)\right)+\eta_{n^{\prime}}^{k}=R_{\tilde{u} n^{\prime}}^{k}\left(\mathbf{P}^{k}\right)+$ $\eta_{n^{\prime}}^{k}, \tilde{R}_{\tilde{u} n^{\prime}}=R_{\tilde{u} n^{\prime}}+\eta_{n^{\prime}}^{k}>\min _{u \in \mathcal{U}_{n^{\prime}}} R_{u n^{\prime}}$. Note that the WSMR of the system is non-decreased, while the power $\left(1-s_{0}^{k}\right) \mathbf{P}_{\mathbf{n}}^{\mathbf{k}}$ is saved from subcarrier $k$ in cell $n \in \mathcal{N}_{2}^{k}$.

Let us introduce $R_{n}^{\min }=\min _{u \in \mathcal{U}_{n}} R_{u n}, \forall n$. After substituting $\mathbf{P}_{n}^{\prime k}$ with $\tilde{\mathbf{P}}_{n}^{k}\left(s_{0}^{k}\right)$, we now have $\forall n \in \mathcal{N}_{2}^{k}, k \in \mathcal{S}_{\tilde{\nu} n}, \tilde{R}_{\tilde{v} n}>$ $R_{n}^{\min }$ and $\forall n \in \mathcal{N}_{1}^{k}, k \in \mathcal{S}_{\tilde{u} n}, \tilde{R}_{\tilde{u} n}>R_{n}^{\min }$. Let us define $\breve{\mathbf{P}}_{n}^{k}\left(c^{k}\right)=c^{k} * \mathbf{P}_{n}^{\prime k}$ where $c^{k} \in(0,1)$. Thus, $\Upsilon_{\mathrm{d}_{u n}, t_{1}}^{k}$ can be denoted as

$$
\frac{P_{\mathrm{s}_{n}, t_{1}}^{\prime k} G_{\mathrm{s}_{n}, \mathrm{~d}_{u n}}^{k}}{\frac{\sigma^{2}}{c^{k}}+\sum_{n^{\prime} \neq n} P_{\mathrm{s}_{n^{\prime}}, t_{1}}^{\prime k} G_{\mathrm{s}_{n^{\prime}}, \mathrm{d}_{u n}}^{k}} .
$$

It is obvious that $\Upsilon_{\mathrm{d}_{u n}, t_{1}}^{k}$ is an increasing function of $c^{k}$. Similarly, $\Upsilon_{\mathrm{d}_{u n}, t_{2}}^{k}, \Gamma_{\mathrm{r}_{n}}^{k}$, and $\Gamma_{\mathrm{d}_{u n}, t_{2}}^{k}$ are also increasing functions of $c^{k}$. Thus, $\breve{R}_{u n}^{k}\left(\breve{\mathbf{P}}^{k}\left(c^{k}\right)\right)$ is an increasing function of $c^{k}$. There exists a $c_{0}^{k} \in(0,1), \alpha_{n}^{k}>0$ such that $\forall n, \breve{R}_{u n}^{k}\left(\breve{\mathbf{P}}^{k}\left(c_{0}^{k}\right)\right)=\breve{R}_{u n}^{k}\left(\breve{\mathbf{P}}^{k}(1)\right)-\alpha_{n}^{k}=R_{u n}^{k}\left(\mathbf{P}^{\prime k}\right)-$ $\alpha_{n}^{k}=\tilde{R}_{u n}^{k}\left(\tilde{\mathbf{P}}^{k}\left(s_{0}^{k}\right)\right)-\alpha_{n}^{k}, \breve{R}_{u n}=\tilde{R}_{u n}-\alpha_{n}^{k}>R_{n}^{\min }$. The power $\left(1-c_{0}^{k}\right) \tilde{\mathbf{P}}_{n}^{k}\left(s_{0}^{k}\right)$ can be saved from subcarrier $k$ in each cell $n$ while keeping the WSMR of the system non-decreased.

We now consider the case when $k$ is in state 2, meaning $n \in \mathcal{N}_{2}^{k}, \forall n$. By using the previous derivations, we can still find $s_{1}^{k} \in(0,1)$, such that the power $\left(1-s_{1}^{k}\right) \mathbf{P}_{\mathbf{n}}^{\mathbf{k}}$ can be saved from subcarrier $k$ in each cell $n$ while keeping the WSMR of the system non-decreased. This concludes the proof of Lemma 1.
We now state the main result of this section. Based on Lemma 1 , the following theorem is proposed, which sheds light on the fairness property of the formulated problem.

Theorem 1. With any feasible values $\mathbf{A}=\mathbf{A}_{0}$ and $\mathbf{B}=$ $\mathbf{B}_{0}$, the optimum power allocation $\mathbf{P}_{\mathrm{opt}}$ of the formulated problem results in the same transmission rates for all users in the same cell.

Proof. When $\mathbf{A}=\mathbf{A}_{0}$ and $\mathbf{B}=\mathbf{B}_{0}$, we assume at the optimum, $\exists n_{0}$ where $\tilde{\mathcal{V}}_{n_{0}} \neq \emptyset$ and a subcarrier $k_{0} \in \mathcal{S}_{\tilde{v} n}$ in state 2 or state 3 . Then, $\forall n, k \in \mathcal{S}_{\tilde{u} n}$ where $\tilde{u} \in \tilde{\mathcal{U}}_{n}$, subcarrier $k$ can be in either state 3 or state 1 .

When subcarrier $k$ is in state 3 , as discussed in the proof of Lemma 1, we can still improve $R_{\tilde{u} n}^{k}$ by decreasing the interfering powers of subcarrier $k$ in cell $n^{\prime}$ while keeping the minimum user rate of cell $n^{\prime}$ non-decreased.

When subcarrier $k$ is in state 1 , as at the optimum $\exists k_{0} \neq$ $k$ that is in state 2 or state 3, we can save power from $\mathbf{P}_{n}^{k_{0}}$ in each cell $n$ while keeping the WSMR of the system nondecreased. Let us denote the saved power in each cell $n$ as $\Delta P_{n}$ and define $\hat{\mathbf{P}}_{n}^{k}\left(t^{k}\right)=t^{k} * \mathbf{P}_{\mathbf{n}}^{\mathbf{k}}$ where $t^{k}>1$. Thus, $\Upsilon_{\mathrm{d}_{\tilde{u} n}, t_{1}}^{k}$ can be denoted as

$$
\frac{P_{\mathrm{s}_{n}, t_{1}}^{k} G_{\mathrm{s}_{n}, \mathrm{~d}_{\tilde{u} n}}^{k}}{\frac{\sigma^{2}}{t^{k}}+\sum_{n^{\prime} \neq n, n^{\prime} \in \mathcal{N}_{1}^{k}} P_{\mathrm{s}_{n^{\prime}}, t_{1}}^{k} G_{\mathrm{s}_{n^{\prime}}, \mathrm{d}_{\tilde{u} n}}^{k}} .
$$

It is obvious that $\Upsilon_{\mathrm{d}_{\tilde{u} n}, t_{1}}^{k}$ is an increasing function of $t^{k}$. Similarly, $\Upsilon_{\mathrm{d}_{\tilde{u}}, t}^{k}, \Gamma_{\mathrm{r}_{n}}^{k}$, and $\Gamma_{\mathrm{d}_{\tilde{u} n}, t_{2}}^{k}$ are also increasing functions of $t^{k}$. Thus, for the subcarrier $k$ in state 1 , $\tilde{R}_{\tilde{u} n}^{k}\left(\hat{\mathbf{P}}^{k}\left(t^{k}\right)\right)$ is an increasing function of $t^{k}$. Thus, there exists a $t_{0}^{k}>1$ such that $\tilde{R}_{\tilde{u} n}^{k}\left(\hat{\mathbf{P}}^{k}\left(t_{0}^{k}\right)\right)>R_{\tilde{u} n}^{k}$ and $\sum_{k \in \hat{\mathcal{K}}}\left(t_{0}^{k}-1\right)\left\|\mathbf{P}_{\mathbf{n}}^{\mathbf{k}}\right\| \leq \Delta P_{n}$. Here, $\hat{\mathcal{K}}$ denotes the set of subcarriers in state 1 .

Therefore, $\forall k \in \mathcal{S}_{\tilde{u} n}$ in cell $n, R_{\tilde{u} n}^{k}$ can still be improved. Thus, the minimum user rate of each cell can still be improved by adjusting power allocation. Specifically, we can find matrices $\mathbf{S}, \mathbf{C}, \mathbf{T}, \mathbf{P}_{1}=\mathbf{S C T P}_{\text {opt }}$, according to the previous discussions, such that $R\left(\mathbf{P}_{1}, \mathbf{A}_{0}, \mathbf{B}_{0}\right)>$ $R\left(\mathbf{P}_{\text {opt }}, \mathbf{A}_{0}, \mathbf{B}_{0}\right)$. Here, each element of $\mathbf{S}, \mathbf{C}$, and $\mathbf{T}$ takes value from $(0,1],(0,1]$, and $[1, \infty)$, respectively. This obviously contradicts the optimum assumption and concludes the proof of Theorem 1 .

\section{Algorithm development}

In this section, we will propose an iterative RA algorithm, which obtains a local optimum of the formulated problem after convergence. The proposed algorithm is based on the coordinate ascent (CA) approach, which divides the whole set of optimization variables into several sets of variables 
and iteratively optimizes each set of variables with other sets of variables fixed.

Specifically, variables $\{\mathbf{P}, \mathbf{A}, \mathbf{B}\}$ are divided into two sets: the integral variable set $\{\mathbf{A}, \mathbf{B}\}$ and the continuous variable set $\{\mathbf{P}\}$. During each iteration, the MSSA stage is first carried out followed by the PA stage. Let us introduce integer $m$ to indicate the iteration number. At the MSSA stage of iteration $m$, the integral variables $\{\mathbf{A}, \mathbf{B}\}$ are optimized with $\mathbf{P}=\mathbf{P}^{m-1}$. While at the PA stage of iteration $m$, the continuous variables $\{\mathbf{P}\}$ are optimized with $\mathbf{A}=\mathbf{A}^{m}$ and $\mathbf{B}=\mathbf{B}^{m}$. Here, a variable with the superscript $m$ denotes the value obtained at the end of iteration $m$. In the following, we first design algorithms for both stages, based on which the iterative RA algorithm will then be stated. Finally, several characteristics of the proposed RA algorithm will be discussed.

\subsection{MSSA optimization}

In this subsection, the MSSA stage for iteration $m$ is considered. After setting $\mathbf{P}$ to $\mathbf{P}^{m-1}$, the formulated problem can be rewritten as

$$
\begin{aligned}
& \max _{\mathbf{A}, \mathbf{B}} R\left(\mathbf{P}^{m-1}, \mathbf{A}, \mathbf{B}\right) \\
& \text { s.t. } C 1, C 2, C 5, C 6, C 7 .
\end{aligned}
$$

Note that each cell has independent constraints for its local integral variables. Therefore problem (20) can be decoupled into $N$ subproblems. As for subproblem $n_{0}$, the minimum rate of all users in the cell $n_{0}$ is maximized subject to the local constraints. Specifically, subproblem $n_{0}$ is formulated as

$$
\begin{aligned}
& \max _{\mathbf{A}_{n_{0}}, \mathbf{B}_{n_{0}}} R_{n_{0}}\left(\mathbf{P}^{m-1}, \mathbf{A}_{n_{0}}, \mathbf{B}_{n_{0}}\right) \\
& \text { s.t. } \\
& C 1^{\prime}: \sum_{u=1}^{U} a_{u n_{0}}^{k}+\sum_{u=1}^{U} b_{u n_{0}}^{k} \leq 1, \forall k, \\
& C 2^{\prime}: a_{u n_{0}}^{k} \in\{0,1\}, b_{u n_{0}}^{k} \in\{0,1\}, \forall u, k, \\
& C 5^{\prime}: P_{s_{n_{0}}, t_{1}}^{k, m-1}\left(1-\sum_{u=1}^{U} a_{u n_{0}}^{k}-\sum_{u=1}^{U} b_{u n_{0}}^{k}\right)=0, \forall k, \\
& C 6^{\prime}: P_{\mathrm{s}_{n_{0}}, t_{2}}^{k, m-1}\left(1-\sum_{u=1}^{U} a_{u n_{0}}^{k}\right)=0, \forall k, \\
& C 7^{\prime}: P_{\mathrm{r}_{n_{0}}}^{k, m-1}\left(1-\sum_{u=1}^{U} b_{u n_{0}}^{k}\right)=0, \forall k .
\end{aligned}
$$

where $\mathbf{A}_{n_{0}}=\left\{a_{u n}^{k} \mid n=n_{0}\right\}, \mathbf{B}_{n_{0}}=\left\{b_{u n}^{k} \mid n=n_{0}\right\}$, and

$$
\begin{aligned}
R_{n_{0}}=\min _{u \in \mathcal{U}_{n_{0}}}( & \sum_{k=1}^{K} a_{u n_{0}}^{k} R_{u n_{0}, 1}^{k}\left(\mathbf{P}^{m-1}\right) \\
& \left.+\sum_{k=1}^{K} b_{u n_{0}}^{k} R_{u n_{0}, 2}^{k}\left(\mathbf{P}^{m-1}\right)\right) .
\end{aligned}
$$

By introducing a slack variable $\xi_{n_{0}}$, problem (21) can be reformulated as

$$
\begin{aligned}
& \max _{\mathbf{A}_{n_{0}}, \mathbf{B}_{n_{0}}, \xi_{n_{0}}} \xi_{n_{0}} \\
& \text { s.t. } C 1^{\prime}, C 2^{\prime}, C 5^{\prime}, C 6^{\prime}, C 7^{\prime} \text { and } \\
& C 3^{\prime}: \sum_{k=1}^{K}\left(a_{u n_{0}}^{k} R_{u n_{0}, 1}^{k}+b_{u n_{0}}^{k} R_{u n_{0}, 2}^{k}\right) \geq \xi_{n_{0}}, \forall u \in \mathcal{U}_{n} .
\end{aligned}
$$

Note that (22) is actually a mixed integer linear program (MILP). Hence, it can be solved by means of typical MILP solvers, like MOSEK and TOMLAB. In order to solve it much faster in polynomial time, we now propose a RRMSSA algorithm and a DR-MSSA algorithm. Specifically, both algorithms consist of three steps: the relaxation step, the rounding step, and the decision step.

During the relaxation step, we first relax constraint $C 2^{\prime}$ as $C 2^{\prime \prime}: a_{u n_{0}}^{k} \in[0,1], b_{u n_{0}}^{k} \in[0,1], \forall u, k$. Then, the MILP is relaxed as a linear program (LP), which can easily be solved by any standard LP algorithm in polynomial time (time that is bounded by a fixed polynomial of the length of the input). Note that as the feasible set of the LP is larger than that of the MILP, the optimal objective function value of the LP is an upper bound of that of the MILP.

During the rounding step, we round the fractional optimal solution $\left(\left\{\mathbf{A}_{n_{0}}^{*}, \mathbf{B}_{n_{0}}^{*}\right\}\right)$ of the LP into an integer solution $\left(\left\{\mathbf{A}_{n_{0}}^{\prime}, \mathbf{B}_{n_{0}}^{\prime}\right\}\right)$. Specifically, each value of the fractional solution is viewed as the probability that this value should be rounded to 1 . Then, a direct rounding (DR) algorithm is $\forall k$,

$$
\begin{aligned}
& a_{u n_{0}}^{k^{\prime}}= \begin{cases}1 & a_{u n_{0}}^{k, *}=\max _{u}\left\{a_{u n_{0}}^{k, *}, b_{u n_{0}}^{k, *}\right\} \\
0 & a_{u n_{0}}^{k, *} \neq \max _{u}\left\{a_{u n_{0}}^{k, *}, b_{u n_{0}}^{k, *}\right\},\end{cases} \\
& b_{u n_{0}}^{k^{\prime}}= \begin{cases}1 & b_{u n_{0}}^{k, *}=\max _{u}\left\{a_{u n_{0}}^{k, *}, b_{u n_{0}}^{k, *}\right\} \\
0 & b_{u n_{0}}^{k, *} \neq \max _{u}\left\{a_{u n_{0}}^{k, *}, b_{u n_{0}}^{k, *}\right\} .\end{cases}
\end{aligned}
$$

After this rounding, the $R_{n_{0}}$ calculated with $\left\{\mathbf{A}_{n_{0}}^{\prime}, \mathbf{B}_{n_{0}}^{\prime}\right\}$ is decreased, compared to that calculated with $\left\{\mathbf{A}_{n_{0}}^{*}, \mathbf{B}_{n_{0}}^{*}\right\}$.

In order to make $R_{n_{0}}\left(\mathbf{P}^{m-1}, \mathbf{A}_{n_{0}}^{\prime}, \mathbf{B}_{n_{0}}^{\prime}\right)$ closer to $R_{n_{0}}\left(\mathbf{P}^{m-1}, \mathbf{A}_{n_{0}}^{*}, \mathbf{B}_{n_{0}}^{*}\right)$, we now use the randomized rounding (RR) algorithm, where $N_{s}$ samples of binary values are generated for variables $\left\{\mathbf{A}_{n_{0}}, \mathbf{B}_{n_{0}}\right\}$ by a randomized process with probability $\left(\left\{\mathbf{A}_{n_{0}}^{*}, \mathbf{B}_{n_{0}}^{*}\right\}\right)$. Each sample is denoted as $\left\{\mathbf{A}_{n_{0}}^{(s)}, \mathbf{B}_{n_{0}}^{(s)}\right\}$. Finally, the feasible sample with the maximum $R_{n_{0}}$ is assigned to $\left\{\mathbf{A}_{n_{0}}^{\prime}, \mathbf{B}_{n_{0}}^{\prime}\right\}$. 
During the decision step, we assign $\left\{\mathbf{A}_{n_{0}}^{m}, \mathbf{B}_{n_{0}}^{m}\right\}$ with

$$
\begin{aligned}
\underset{\mathbf{A}_{n_{0}}, \mathbf{B}_{n_{0}}}{\operatorname{argmax}} & \left(R_{n_{0}}\left(\mathbf{P}^{m-1}, \mathbf{A}_{n_{0}}^{\prime}, \mathbf{B}_{n_{0}}^{\prime}\right),\right. \\
& \left.R_{n_{0}}\left(\mathbf{P}^{m-1}, \mathbf{A}_{n_{0}}^{m-1}, \mathbf{B}_{n_{0}}^{m-1}\right)\right) .
\end{aligned}
$$

\subsection{PA optimization}

In this subsection, the PA stage for iteration $m$ is considered. After setting the indicators to $\left\{\mathbf{A}^{m}, \mathbf{B}^{m}\right\}$, the transmission modes are fixed in all cells. Let us denote by $\mathcal{S}_{\text {un }}(d)\left(\mathcal{S}_{\text {un }}(r)\right)$ the set of subcarriers allocated to MS $d_{u n}$ in direct (relay-aided) mode. Then, the objective function of problem (12) can be rewritten as $R\left(\mathbf{P}, \mathbf{A}^{m}, \mathbf{B}^{m}\right)$, given by

$$
\begin{aligned}
\sum_{n} \omega_{n} \min _{u \in \mathcal{U}_{n}} & \left(\sum_{k \in \mathcal{S}_{u n}(d)}\left(\ln \left(1+\Upsilon_{\mathrm{d}_{u n}, t_{1}}^{k}\right)+\ln \left(1+\Upsilon_{\mathrm{d}_{u n}, t_{2}}^{k}\right)\right)\right. \\
& \left.+\sum_{k \in \mathcal{S}_{u n}(r)} \ln \min \left(1+\Gamma_{\mathrm{r}_{n}}^{k}, 1+\Gamma_{\mathrm{d}_{u n, t_{2}}}^{k}\right)\right) .
\end{aligned}
$$

Note that $R$ is a non-convex function due to the presence of interfering power terms in the denominators of $\Upsilon_{\mathrm{d}_{u n}, t_{1}}^{k}, \Upsilon_{\mathrm{d}_{u n}, t_{2}}^{k}, \Gamma_{\mathrm{r}_{n}}^{k}$, and $\Gamma_{\mathrm{d}_{u n, t_{2}}}^{k}$. To solve problem (12), we first replace it with an equivalent complementary geometric program (CGP; see Additional file 1) that is then addressed by means of the algorithm based on SCGP. Note that this methodology has also been used in [26] to tackle another optimization problem.

The equivalent CGP is obtained by formulating problem (12) as a minimization problem. Problem (12) is first converted into an equivalent one given by

$$
\begin{aligned}
& \min _{\mathbf{P}} e^{-R\left(\mathbf{P}, \mathbf{A}^{m}, \mathbf{B}^{m}\right)} \\
& \text { s.t. } C 3-C 7,
\end{aligned}
$$

where $e^{-R\left(\mathbf{P}, \mathbf{A}^{m}, \mathbf{B}^{m}\right)}$ is given by

$$
\begin{gathered}
\prod_{n} \max _{u \in \mathcal{U}_{n}}\left(\prod_{k \in \mathcal{S}_{u n}(d)} \frac{1}{1+\Upsilon_{\mathrm{d}_{u n}, t_{1}}^{k}} \frac{1}{1+\Upsilon_{\mathrm{d}_{u n}, t_{2}}^{k}}\right. \\
\left.\prod_{k \in \mathcal{S}_{u n}(r)} \max \left(\frac{1}{1+\Gamma_{\mathrm{r}_{n}}^{k}}, \frac{1}{1+\Gamma_{\mathrm{d}_{u n, t_{2}}}^{k}}\right)\right)^{\omega_{n}},
\end{gathered}
$$

and

$$
\frac{1}{1+\Upsilon_{\mathrm{d}_{u n}, t_{1}}^{k}}=\frac{f_{\mathrm{d}_{u n}, t_{1}}^{k}}{f_{\mathrm{d}_{u n}, t_{1}}^{k}+P_{\mathrm{s}_{n}, t_{1}}^{k} G_{\mathrm{s}_{n}, \mathrm{~d}_{u n}}^{k}}
$$

$$
\begin{aligned}
& \frac{1}{1+\Upsilon_{\mathrm{d}_{u n}, t_{2}}^{k}}=\frac{f_{\mathrm{d}_{u n}, t_{2}}^{k}}{f_{\mathrm{d}_{u n}, t_{2}}^{k}+P_{\mathrm{s}_{n}, t_{2}}^{k} G_{\mathrm{s}_{n}, \mathrm{~d}_{u n}}^{k}} \\
& \frac{1}{1+\Gamma_{\mathrm{d}_{u n}, t_{2}}^{k}}=\frac{f_{\mathrm{d}_{u n}, t_{2}}^{k}}{f_{\mathrm{d}_{u n}, t_{2}}^{k}+P_{\mathrm{r}_{n}}^{k} G_{\mathrm{r}_{n}, \mathrm{~d}_{u n}}^{k}} \\
& \frac{1}{1+\Gamma_{\mathrm{r}_{n}}^{k}}=\frac{f_{\mathrm{r}_{n}}^{k}}{f_{\mathrm{r}_{n}}^{k}+P_{\mathrm{s}_{n}, t_{1}}^{k} G_{\mathrm{s}_{n}, \mathrm{r}_{n}}^{k}} .
\end{aligned}
$$

Second, another equivalent formulation is obtained by introducing slack variables $\left\{R_{n}^{\prime}\right\}$ and $\Psi_{r}=$ $\left\{\Psi_{r}^{u n k}, \forall u, n, k \in \mathcal{S}_{u n}(r)\right\}$. Then, problem (24) can be formulated as

$$
\min _{\mathbf{P},\left\{R_{n}^{\prime}\right\}, \Psi_{r}} \prod_{n} R_{n}^{\prime}
$$

s.t. $C 3-C 7$,

$C 8: \frac{1}{R_{n}^{\prime}}\left(\prod_{k \in \mathcal{S}_{u n}(d)} \frac{f_{\mathrm{d}_{u n}, t_{1}}^{k}}{g_{\mathrm{d}_{u n}, t_{1}}^{k}} \frac{f_{\mathrm{d}_{u n}, t_{1}}^{k}}{g_{\mathrm{d}_{u n}, t_{2}}^{\prime k}} \prod_{k \in \mathcal{S}_{u n}(r)} \Psi_{r}^{u n k}\right)^{\omega_{n}} \leq 1, \forall u, n$,

C9 : $\frac{f_{\mathrm{r}_{n}}^{k}}{g_{\mathrm{r}_{n}}^{k} \Psi_{r}^{u n k}} \leq 1, \forall u, n, k \in \mathcal{S}_{u n}(r)$,

$C 10: \frac{f_{\mathrm{d}_{u n}, t_{2}}^{k}}{g_{\mathrm{d}_{u n}, t_{2}}^{k} \Psi_{r}^{u n k}} \leq 1, \forall u, n, k \in \mathcal{S}_{u n}(r)$,

where

$$
\begin{aligned}
& g_{\mathrm{d}_{u n}, t_{1}}^{k}=f_{\mathrm{d}_{u n}, t_{1}}^{k}+P_{\mathrm{s}_{n}, t_{1}}^{k} G_{\mathrm{s}_{n}, \mathrm{~d}_{u n}}^{k}, \\
& g_{\mathrm{d}_{u n}, t_{2}}^{k}=f_{\mathrm{d}_{u n}, t_{2}}^{k}+P_{\mathrm{s}_{n}, t_{2}}^{k} G_{\mathrm{s}_{n}, \mathrm{~d}_{u n}}^{k}, \\
& g_{\mathrm{d}_{u n}, t_{2}}^{k}=f_{\mathrm{d}_{u n}, t_{2}}^{k}+P_{\mathrm{r}_{n}}^{k} G_{\mathrm{r}_{n}, \mathrm{~d}_{u n}}^{k}, \\
& g_{\mathrm{r}_{n}}^{k}=f_{\mathrm{r}_{n}}^{k}+P_{\mathrm{s}_{n}, t_{1}}^{k} G_{\mathrm{s}_{n}, \mathrm{r}_{n}}^{k} .
\end{aligned}
$$

Problem (30) consists of an objective function and bounding constraints which all are ratios of two posynomials, making the problem belong to the class of CGP [31]. Problem (30) cannot be made convex and is NP-hard [32]. In order to solve it, the SCGP algorithm is now proposed. The proposed SCGP algorithm approximates the non-convex problem (30) into a series of standard GPs. Therefore, it belongs to the class of successive convex approximation methods [33]. The SCGP is described in Algorithm 1. Integer $m^{\prime}$ indicates the current iteration number, and a variable with superscripts $m$ and $m^{\prime}$ denotes the value obtained at the end of inner iteration $m^{\prime}$ in outer iteration $m$. 


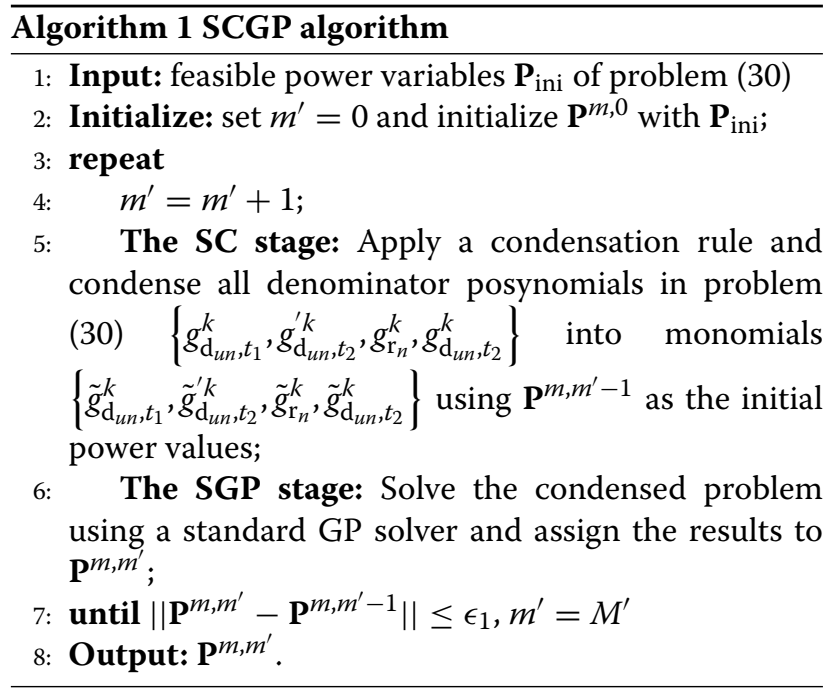

Specifically in the proposed SCGP algorithm, we first set $m^{\prime}=0$ and initialize the PA vector $\mathbf{P}^{m, 0}$ with $\mathbf{P}_{\text {ini }}$. Each iteration $m^{\prime}$ contains an SC stage followed by a standard geometric programming (SGP) stage.

During the SC stage, a GP approximation of problem (30) is constructed by using the method of Lemma 1 in [31] to condense all denominator posynomials $\quad\left\{g_{\mathrm{d}_{u n}, t_{1}}^{k}, g_{\mathrm{d}_{u n}, t_{2}}^{\prime k}, g_{\mathrm{r}_{n}}^{k}, g_{\mathrm{d}_{u n}, t_{2}}^{k}\right\} \quad$ into monomials $\left\{\tilde{g}_{\mathrm{d}_{u n}, t_{1}}^{k}, \tilde{g}_{\mathrm{d}_{u n}, t_{2}}^{k}, \tilde{g}_{\mathrm{r}_{\mathrm{r}}}^{k}, \tilde{g}_{\mathrm{d}_{u n}, t_{2}}^{k}\right\}$. Specifically, the exact expressions of the monomials are given in Additional file 2. Functions $h_{1}^{n, k}=\frac{f_{\mathrm{r}_{n}}^{k}}{g_{\mathrm{r}_{n}}^{k} \Psi_{r}^{u n k}}, h_{2}^{n, k}=\frac{f_{\mathrm{d}_{u n}, t_{2}}^{k}}{g_{\mathrm{d}_{u n}, t_{2}} \Psi_{r}^{u n k}}$ and $h_{3}^{n, k}=$ $\frac{1}{R_{n}^{\prime}}\left(\prod_{k \in \mathcal{S}_{u n}(d)} \frac{f_{\mathrm{d}_{u n}, t_{1}}^{k}}{g_{\mathrm{d}_{u n}, t_{1}}^{k}} \frac{f_{\mathrm{d}_{u n}, t_{1}}^{k}}{g_{\mathrm{d}_{u n}, t_{2}}^{\prime k}} \prod_{k \in \mathcal{S}_{u n}(r)} \Psi_{r}^{u n k}\right)^{\omega_{n}}$ are approximated by, respectively, $\tilde{h}_{1}^{n, k}=\frac{f_{\mathrm{r} n}^{k}}{\tilde{g}_{r_{n}}^{k} \Psi_{r}^{u n k}}, \tilde{h}_{2}^{n, k}=\frac{f_{\mathrm{d}_{u n}, t_{2}}^{k}}{\tilde{g}_{\mathrm{d}_{u n}, t_{2}}^{k} \Psi_{r}^{u n k}}$ and $\tilde{h}_{3}^{n, k}=\frac{1}{R_{n}^{\prime}}\left(\prod_{k \in \mathcal{S}_{u n}(d)} \frac{f_{\mathrm{d}_{u n}, t_{1}}^{k}}{\tilde{g}_{\mathrm{d}_{u n}, t_{1}}^{k}} \frac{f_{\mathrm{d}_{u n}, t_{1}}^{k}}{\tilde{g}_{\mathrm{d}_{u n}, t_{2}}^{\prime k}} \prod_{k \in \mathcal{S}_{\mathcal{L}_{n}(r)}} \Psi_{r}^{u n k}\right)^{\omega_{n}}$. According to proposition 3 in [31], all the approximations satisfy the three conditions proposed in [33] for the convergence of the successive approximation method. By denoting $\Theta=\left\{\mathbf{P},\left\{R_{n}^{\prime}\right\}, \Psi_{r}\right\}$, the three conditions are listed as follows:

1. Bounding condition: $\forall \Theta$,

$$
h_{i}^{n, k}(\Theta) \leq \tilde{h}_{i}^{n, k}(\Theta), \forall i=1,2,3 .
$$

2. Tightness condition: At the beginning of iteration $m^{\prime}$,

$$
h_{i}^{n, k}\left(\Theta^{m, m^{\prime}-1}\right)=\tilde{h}_{i}^{n, k}\left(\Theta^{m, m^{\prime}-1}\right), \forall i=1,2,3 .
$$

3. Differential condition: At the beginning of iteration $m^{\prime}, \forall \theta \in \Theta$,

$$
\frac{\partial h_{i}^{n, k}\left(\Theta^{m, m^{\prime}-1}\right)}{\partial \theta}=\frac{\partial \tilde{h}_{i}^{n, k}\left(\Theta^{m, m^{\prime}-1}\right)}{\partial \theta}, \forall i=1,2,3 .
$$

After the SC stage, problem (30) is formulated by a standard GP. The SGP stage amounts to solving the GP by means of a standard GP solver, e.g., the software provided in [34]. The output provided by this stage corresponds to $\mathbf{P}^{m, m^{\prime}}$

Thanks to the three conditions fulfilled during the SC stage, our proposed SCGP algorithm is a general inner approximation algorithm [33], which will converge to a local optimum satisfying the KKT conditions of problem (30) according to corollary 1 of [33]. In practice, the iterations of the SCGP algorithm will be stopped when $\left\|\mathbf{P}^{m, m^{\prime}}-\mathbf{P}^{m, m^{\prime}-1}\right\| \leq \epsilon_{1}$ or when $m^{\prime}$ exceeds a prescribed value $M^{\prime}$.

\subsection{The proposed RA algorithm and its characteristics}

Based on the algorithms proposed at both the MSSA stage and the PA stage, we now propose the overall RA algorithm as follows:

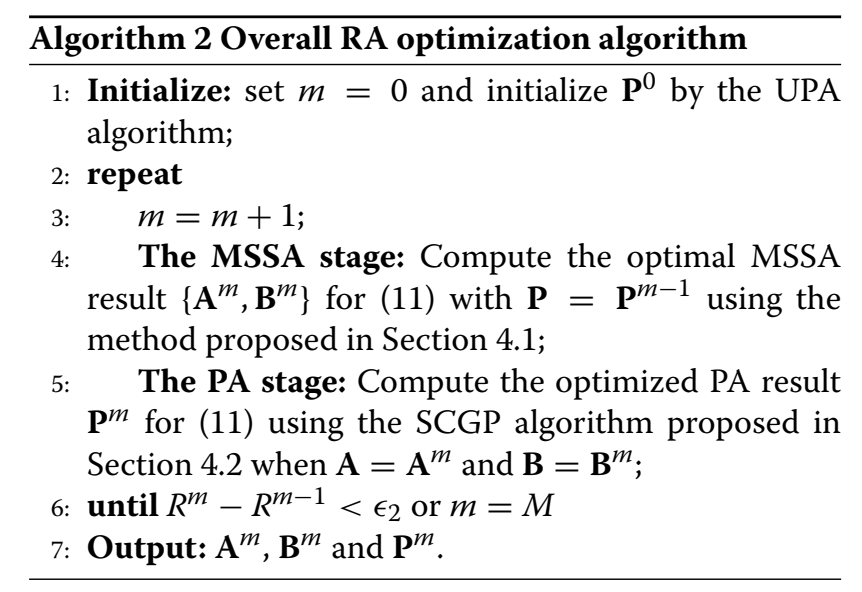

Specifically in the proposed RA algorithm, we first set $m=0$ and initialize the power $\mathbf{P}^{0}$ by uniform power allocation (UPA). Each iteration consists of the MSSA stage and the PA stage. During the MSSA stage of iteration $m$, we set $\mathbf{P}=\mathbf{P}^{m-1}$ and decouple problem (12) into $n$ MILPs, which can be solved by either typical MILP solvers (named the MSSA algorithm) or the RR-MSSA algorithm and the DR-MSSA algorithm proposed in Section 4.1. Note that both the RR-MSSA algorithm and the DRMSSA algorithm can be solved in polynomial time. The output delivered is denoted as $\mathbf{A}^{m}$ and $\mathbf{B}^{m}$. Finally, we have $R\left(\mathbf{P}^{m-1}, \mathbf{A}^{m-1}, \mathbf{B}^{m-1}\right) \leq R\left(\mathbf{P}^{m-1}, \mathbf{A}^{m}, \mathbf{B}^{m}\right)$. 
During the PA stage of iteration $m$, we set $\mathbf{A}=\mathbf{A}^{m}$, $\mathbf{B}=\mathbf{B}^{m}$ and solve problem (12) using the SCGP algorithm proposed in Section 4.1. The output delivered is denoted as $\mathbf{P}^{m}$. As shown in Section 4.2, the solutions of the approximated GP problems converge to a local optimum satisfying the Karush-Kuhn-Tucker (KKT) conditions of the non-convex problem. Note that as each standard GP can be solved in polynomial time [32], $\mathbf{P}^{m}$ will be obtained in polynomial time after the convergence of the SCGP algorithm. Finally, we have $R\left(\mathbf{P}^{m-1}, \mathbf{A}^{m}, \mathbf{B}^{m}\right) \leq$ $R\left(\mathbf{P}^{m}, \mathbf{A}^{m}, \mathbf{B}^{m}\right)$

Considering the proposed RA algorithm, we now have

$$
\begin{aligned}
& R\left(\mathbf{P}^{m-1}, \mathbf{A}^{m-1}, \mathbf{B}^{m-1}\right) \\
E 1: \leq & R\left(\mathbf{P}^{m-1}, \mathbf{A}^{m}, \mathbf{B}^{m}\right) \\
E 2: \leq & R\left(\mathbf{P}^{m}, \mathbf{A}^{m}, \mathbf{B}^{m}\right)
\end{aligned}
$$

where E1 and E2 are due to effects of the MSSA stage and the PA stage, respectively. This means that Algorithm 2 yields non-decreasing sum rates with iterations. Moreover, the optimum sum rate is upper bounded due to the total power constraint in each cell. Thus, the sum rate values will not increase indefinitely with iterations, meaning that the iterations will eventually converge. Algorithm 2 will stop when the sum rate increase is below a prescribed value $\epsilon_{2}$ or when $m$ reaches a prescribed value $M$.

At the end of iteration $m$, a local optimum of the formulated problem is obtained at the SCGP stage, which is then improved at the MSSA stage of the next iteration. After that, a better local optimum can be calculated at the SCGP stage of iteration $m+1$. Finally, a good local optimum can be obtained after convergence. As both problems at two stages can be solved by the proposed algorithms in polynomial time, the formulated problem can be solved by Algorithm 2 in polynomial time.

We now propose Theorem 2 as follows:

Theorem 2. After the convergence of Algorithm 2, per-cell user fairness is reached, meaning that every user in the same cell obtains the same transmission rate.

Proof. As mentioned before, after the convergence of Algorithm 2, a local optimum of problem (12) is obtained. Let us denote the optimal solutions as $\mathbf{A}^{*}, \mathbf{B}^{*}$, and $\mathbf{P}^{*}$. Thus, for the local optimum, there exists $r>0$, such that $\forall \mathbf{P} \in \mathbf{B}_{r}\left(\mathbf{P}^{*}\right), R\left(\mathbf{P}, \mathbf{A}^{*}, \mathbf{B}^{*}\right) \leq R\left(\mathbf{P}^{*}, \mathbf{A}^{*}, \mathbf{B}^{*}\right)$. Here, $\mathbf{B}_{r}\left(\mathbf{P}^{*}\right)=\left\{\mathbf{P} \mid d\left(\mathbf{P}, \mathbf{P}^{*}\right)<r\right\}$ is the open ball with center $\mathbf{P}^{*}$ and radius $r$, where $d\left(\mathbf{P}, \mathbf{P}^{*}\right)$ denotes the Euclidean distance between $\mathbf{P}$ and $\mathbf{P}^{*}$. Let us assume $\exists n$ where $\tilde{\mathcal{V}}_{n} \neq \emptyset$. Following the methodology similar to the proof of Theorem 1, there exists matrices $\mathbf{S}, \mathbf{C}, \mathbf{T}$ and $\mathbf{P}_{1}=\mathbf{S C T P}^{*} \in \mathbf{B}_{r}\left(\mathbf{P}^{*}\right)$, such that $R\left(\mathbf{P}_{1}, \mathbf{A}^{*}, \mathbf{B}^{*}\right)>$ $R\left(\mathbf{P}^{*}, \mathbf{A}^{*}, \mathbf{B}^{*}\right)$. Here, each element of $\mathbf{S}, \mathbf{C}$, and $\mathbf{T}$ takes the value from $(0,1],(0,1]$, and $[1, \infty)$, respectively. This obviously contradicts the previous optimality statement, and $\tilde{\mathcal{V}}_{n}=\emptyset$, which concludes the proof of Theorem 2 .

\section{Numerical experiments}

In this section, the performance of our proposed RA algorithm as well as the benefits of using OR and the HSE protocol will be discussed. To illustrate the convergence and effectiveness of the algorithm, the rates of all users and the WSMRs are first presented for one particular channel realization. Then, the results are provided and discussed for the performance of the algorithms averaged over many channel realizations. Note that we introduce the MSSA-based algorithm, the RR-MSSA-based algorithm, and the DR-MSSA-based algorithm to denote the proposed iterative RA algorithms which adopt the MSSA algorithm, the RR-MSSA algorithm, and the DRMSSA algorithm, respectively, at the MSSA stage.

\subsection{System setup}

For the purpose of illustration, a multi-cell OFDMA system with $N=3$ coordinated cells and $K=32$ available subcarriers is considered. Each cell contains $U=4 \mathrm{MSs}$. Figure 1 shows the positions of the BSs, the RSs, and the MSs expressed in meters. Specifically, the coordinates of BS 1,2 , and 3 are $(-100 \sqrt{3},-100),(100 \sqrt{3},-100)$, and $(0,200)$, respectively. Also, the coordinates of RS 1 , 2 , and 3 are $(-100 \sqrt{3},-60),(100 \sqrt{3},-60)$, and $(0,160)$, respectively. Each member of the MS group $\mathcal{U}_{n}$ in cell $n$ is located randomly and uniformly inside a confined region of that cell, which is depicted as a blue box in Figure 1.

The channel impulse response (CIR) of each link is drawn randomly from an 8-tap delay line model, where each tap has a circular complex Gaussian distribution with zero mean and variance as $\sigma_{i}^{2}$. We further assume $\frac{\sigma_{i}^{2}}{\sigma_{i+1}^{2}}=$

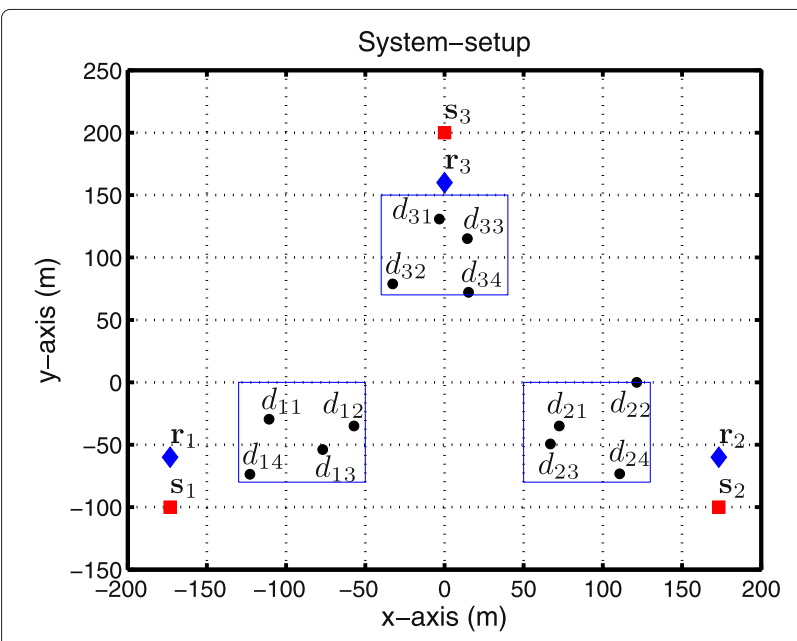

Figure 1 A random system setup. 
$e^{3}$, meaning that the tap power decreases exponentially with a coefficient 3 . Moreover, $\sum_{i} \sigma_{i}^{2}=d^{-3}$, meaning that the received power decreases exponentially with distance $d$ and the propagation exponent equals 3. Finally, the CFR of each link is computed from its CIR using the $K$-point FFT. Each cell is assumed to have the same total power constraint, and we set $\omega_{n}=1, P_{t, n}=P_{t}, \forall n$, $\sigma^{2}=-70 \mathrm{dBm}, \epsilon_{1}=R_{\text {ini }} / 100$, and $\epsilon_{2}=R_{\text {ini }} / 100$. Here, $R_{\text {ini }}$ denotes the sum rate calculated with the initial RA results at the beginning of each iteration.

\subsection{Results for a random realization of channels}

In order to illustrate the convergence and effectiveness of three RA algorithms, we set $P_{t}=20 \mathrm{dBm}$ and $U=4$. The positions of MSs and a set of channels are randomly generated. Specifically, the coordinates of $d_{11}, d_{12}, d_{13}, d_{14}, d_{21}, d_{22}, d_{23}, d_{24}, d_{31}, d_{32}, d_{33}$, and $d_{34}$ are $(-110.9,-29.6),(-57,-34.9),(-76.9,-53.9)$, $(-122.9,-73.6),(72.5,-35),(121.4,-0.1),(66.9,-49.5)$, (110.6, -73.4), (-3.2, 130.8), (-32.6, 78.9), (14.6, 115.1), and $(15.2,72.2)$, respectively.

As shown in Figure 2, using the three proposed algorithms, the WSMRs keep increasing continuously and converge smoothly to the final rate. After convergence, the WSMRs are increased by around $74 \%, 73 \%$, and $55 \%$ using the MSSA-based algorithm, the RR-MSSA-based algorithm, and the DR-MSSA-based algorithm, respectively. Compared to the MSSA-based algorithm, the RR-MSSAbased algorithm has similar performance and less complexity. Thus, it is preferred for practical implementation.

Figure 3 shows the optimized user rates in three cells using three RA algorithms. After the convergence of each RA algorithm, the minimal user rate in each cell is enhanced and all users in the same cell obtain the same rates. Therefore, the fairness of our algorithms is illustrated.

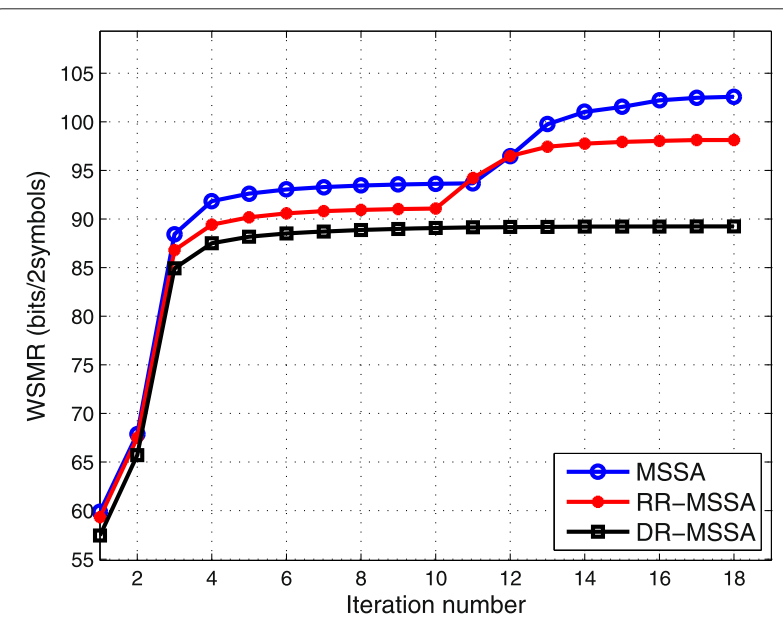

Figure 2 Convergence of the WSMR.
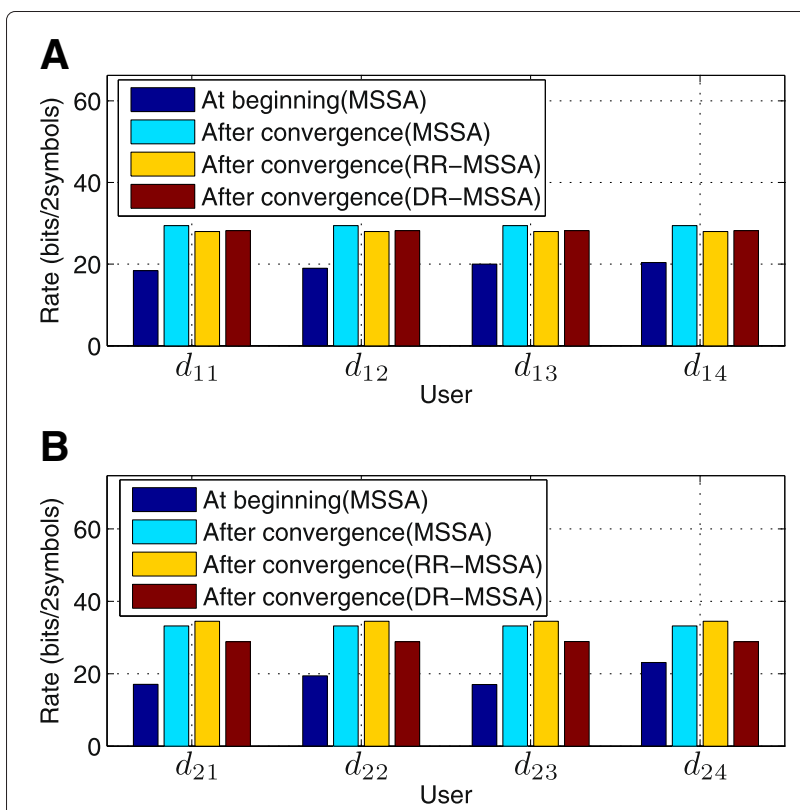

C

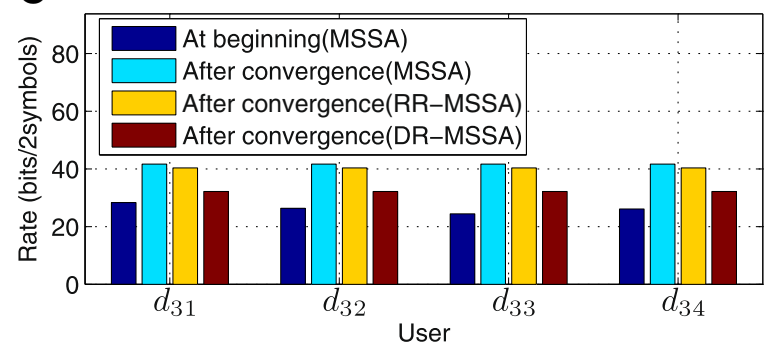

Figure 3 Rate results for the random realization of channels. Rates for users in (A) cell 1, (B) cell 2, and (C) cell 3.

\subsection{Results averaged over channel distribution}

In order to illustrate the average performance of our proposed RA algorithms, 100 random realizations of channels are generated with $U=8$. We set up different total power constraints ranging from 0 to $50 \mathrm{dBm}$. To show the benefit of using OR and the HSE protocol, we now compare the proposed protocol with a FR protocol and a LSE protocol. Specifically, the FR protocol is the same as the proposed protocol except that all subcarriers are forced to be in relay-aided mode. The LSE protocol is also the same as the proposed protocol except that all direct mode subcarriers are activated only during the first TS. We also compare the performances of the MSSA-based algorithm, the RR-MSSA-based algorithm, and the DR-MSSA-based algorithm.

Figure 4 adopts the MSSA-based algorithm. It appears that the average WSMR is increased significantly after optimization, especially when $P_{t}$ takes high values. This confirms the effectiveness of our proposed algorithm. Figure 5 provides the average WSMRs for the FR protocol, the LSE protocol, and the proposed protocol. We can 


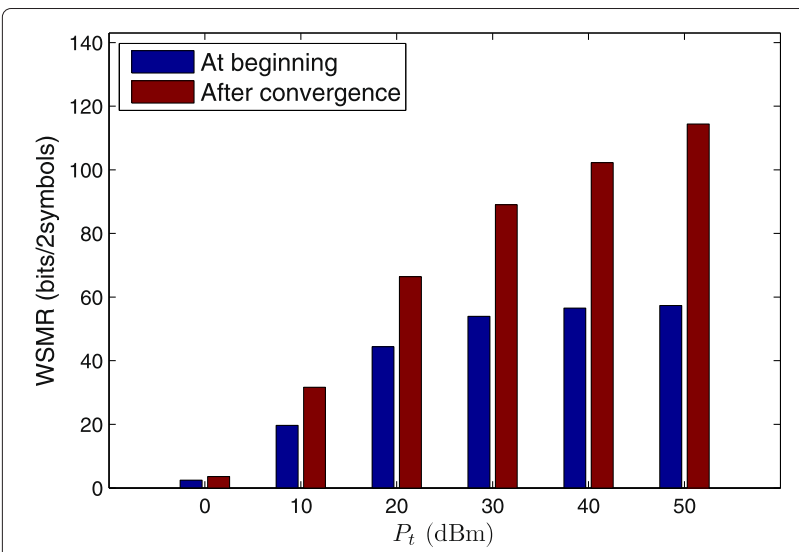

Figure 4 Average WSMRs over 100 random realizations of channels.

see that the proposed protocol performs much better than the other two protocols. Thus, the effectiveness of using OR and the HSE protocol is illustrated. Figure 6 provides the average WSMRs of using the MSSA-based algorithm, the RR-MSSA-based algorithm, and the DR-MSSA-based algorithm. We can see that the RR-MSSA-based algorithm performs similarly as the MSSA-based algorithm and better than the DR-MSSA-based algorithm. Note that the RR-MSSA-based algorithm can be solved in polynomial time. Thus, it offers a good trade-off between the performance and the complexity.

\section{Conclusion}

We have considered a multi-cell OFDMA downlink system where the BS transmissions are aided by a DF RS with OR and the HSE protocol. Assuming a central controller, we have formulated the WSMR maximization problem with per-cell total power constraints. Three iterative RA algorithms have been proposed, and their convergence as well as fairness characteristics were proven. Through

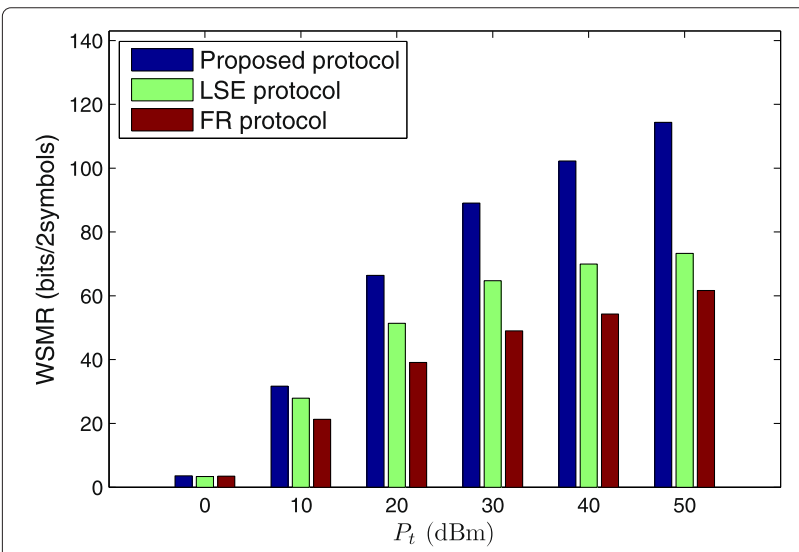

Figure 5 Comparison of average WSMRs for three protocols.

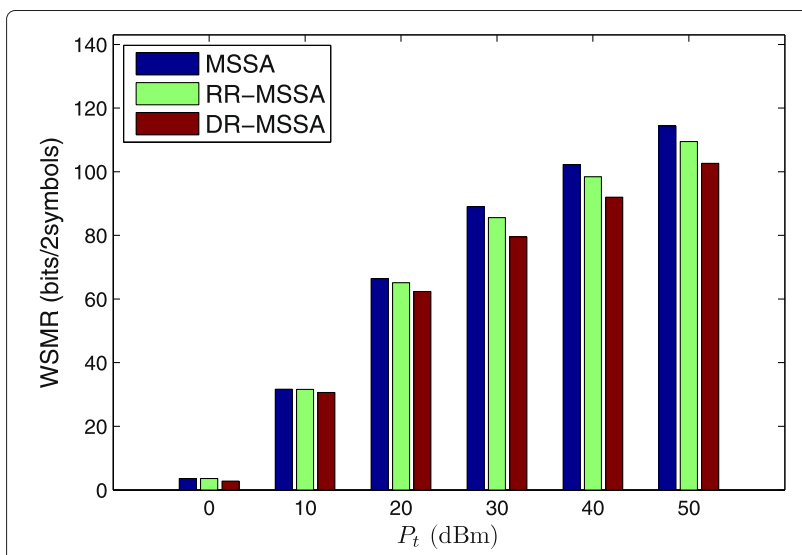

Figure 6 Comparison of average WSMRs with three RA algorithms.

numerical experiments, the convergence and effectiveness of the proposed algorithms as well as the benefit of using OR and the HSE protocol have been illustrated. Future work will be devoted to considering signal combining schemes at the destinations and AF/compress-andforward (CF) relaying protocols with subcarrier pairing. Moreover, the case of multiple RSs and individual power constraints will also be investigated.

\section{Additional files}

Additional file 1: Complementary GP [31,35]. In this file, the definition and some properties of the complementary GP are given.

Additional file 2: Exact expressions of the condensed monomials in Section 4.2. In this file, exact expressions of the condensed monomials in Section 4.2 are given.

\section{Abbreviations}

AWGN: additive white Gaussian noise; AF: amplify and forward; BS: base station; CCl: cochannel interference; CF: compress-and-forward; CFR: channel frequency response; CGP: complementary geometric program; CIR: channel impulse response; CSI: channel state information; DF: decode and forward; DR: direct rounding; DR-MSSA: direct rounding-based MSSA; GP: geometric program; HSE: high spectrum efficiency; KKT: Karush-Kuhn-Tucker; LSE: Iow spectrum efficiency; MILPs: mixed integer linear programs; MSSA: mode selection and subcarrier assignment; MS: mobile station; OFDM: orthogonal frequency division modulation; OFDMA: orthogonal frequency division multiple access; OR: opportunistic relaying; PA: power allocation; RA: resource allocation; RR: randomized rounding; RR-MSSA: randomized rounding-based MSSA; RS: relay station; SCGP: single condensation and geometric programming; SGP: standard geometric programming; SINR: signal-to-interference-plus-noise ratio; TS: time slot; WSMR: weighted sum of per cell min-rate.

\section{Competing interests}

The authors declare that they have no competing interests.

\section{Acknowledgments}

The authors would like to thank Prof. S. Boyd for sharing the GGPLAB toolbox online, the China Scholarship Council (CSC) for funding this work, and the Belgian Science Policy Office (BELSPO) for the support of the Inter-university Attractive Poles Programme (IAP) BESTCOM network. The work of T. Wang is supported by the Program for Professor of Special Appointment (Eastern Scholar) at Shanghai Institutions of Higher Learning. 


\section{Author details}

${ }^{1}$ ICTEAM institute (École Polytechnique de Louvain), Université catholique de Louvain, Louvain-la-Neuve 1348, Belgium. ${ }^{2}$ School of Communication and Information Engineering, Shanghai University, Shanghai 200072, China.

${ }^{3}$ School of Electronic Science and Engineering, National University of Defense Technology, Changsha 410073, China.

\section{Received: 27 November 2013 Accepted: 31 March 2014}

\section{Published: 1 May 2014}

\section{References}

1. R Pabst, BH Walke, DC Schultz, P Herhold, H Yanikomeroglu, S Mukherjee, H Viswanathan, M Lott, W Zirwas, M Dohler, H Aghvami, DD Falconer, GP Fettweis, Relay-based deployment concepts for wireless and mobile broadband radio. IEEE Commun. Mag. 42(9), 80-89 (2004)

2. JN Laneman, GW Wornell, Distributed space-time-coded protocols for exploiting cooperative diversity in wireless networks. IEEE Trans. Inform. Theor. 49(10), 2415-242 (2003)

3. JN Laneman, DNC Tse, GW Wornell, Cooperative diversity in wireless networks: efficient protocols and outage behavior. IEEE. Trans. Inform. Theor. 50(12), 3062-3080 (2004)

4. W Wang, S Yan, S Yang, Optimally joint subcarrier matching and power allocation in OFDM multihop system. Eurasip J. Adv. Signal Process. 2008, 1-8 (2008)

5. W Wang, R Wu, Capacity maximization for OFDM two-hop relay system with separate power constraints. IEEE Trans. Veh. Tech. 58(9), 4943-4954 (2009)

6. Y Li, W Wang, J Kong, M Peng, Subcarrier pairing for amplify-and-forward and decode-and-forward OFDM relay links. IEEE Comm. Lett. 13(4), 209-211 (2009)

7. TWang, Weighted sum power minimisation for multichannel decode-and-forward relaying. IET Electron. Lett. 48(7), 410-411 (2012)

8. L Vandendorpe, RT Duran, J Louveaux, A Zaidi, Power allocation for OFDM transmission with DF relaying, in IEEE International Conference on Communications (IEEE, Beijing, 2008), pp. 3795-3800

9. J Louveaux, RT Duran, L Vandendorpe, Efficient algorithm for optimal power allocation in OFDM transmission with relaying, in IEEE ICASSP 2008 (IEEE, Las Vegas, 2008), pp. 3257-3260

10. TCY Ng, W Yu, Joint optimization of relay strategies and resource allocations in cooperative cellular networks. IEEE J. Sel. Area Comm. 25(2), 328-339 (2007)

11. W Ying, Q Xin-chun, W Tong, L Bao-ling, Power allocation and subcarrier pairing algorithm for regenerative OFDM relay system, in IEEE VTC2007-Spring (IEEE Dublin, 2007), pp. 2727-2731

12. Y Li, W Wang, J Kong, W Hong, X Zhang, M Peng, Power allocation and subcarrier pairing in OFDM-based relaying networks, in IEEE International Conference on Communications, 2008 (IEEE, Beijing, 2008), pp. 2602-2606

13. M Hajiaghayi, M Dong, B Liang, Optimal channel assignment and power allocation for dual-hop multi-channel multi-user relaying, in IEEE INFOCOM 2011 (IEEE, Shanghai, 2011), pp. 76-80

14. L Vandendorpe, J Louveaux, O Oguz, A Zaidi, Improved OFDM transmission with DF relaying and power allocation for a sum power constraint, in 3rd International Symposium on Wireless Pervasive Computing (IEEE, Santorini, 2008), pp. 665-669

15. L Vandendorpe, J Louveaux, O Oguz, A Zaidi, Power allocation for improved DF relayed OFDM transmission: the individual power constraint case, in IEEE International Conference on Communications (IEEE, Dresden, 2009), pp. 1-6

16. L Vandendorpe, J Louveaux, O Oguz, A Zaidi, Rate-optimized power allocation for DF-relayed OFDM transmission under sum and individual power constraints. EURASIP J. Wirel. Commun. Netw. 2009(11), 1-11 (2009)

17. C-N Hsu, H-J Su, P-H Lin, Joint subcarrier pairing and power allocation for OFDM transmission with decode-and-forward relaying. IEEE Trans. Signal Process. 59(1), 399-414 (2011)

18. T Wang, L Vandendorpe, Wsr maximized resource allocation in multiple DF relays aided OFDMA downlink transmission. IEEE Trans. Signal Process. 59(8), 3964-3976 (2011)

19. TWang, L Vandendorpe, Sum rate maximized resource allocation in multiple DF relays aided OFDM transmission. IEEE J. Sel. Area Comm. 29(8), 1559-1571 (2011)
20. H Boostanimehr, VK Bhargava, Selective subcarrier pairing and power allocation for DF OFDM relay systems with perfect and partial CSI. IEEE Trans. Wireless Comm. 10(12), 4057-4067 (2011)

21. Y Liu, M Tao, An optimal graph approach for optimizing OFDMA relay networks, in 2012 IEEE ICC (IEEE, Ottawa, 2012), pp. 4277-4281

22. T Wang, Y Fang, L Vandendorpe, Power minimization for OFDM transmission with subcarrier-pair based opportunistic DF relaying. IEEE Comm. Lett. 17(3), 471-474 (2013)

23. TWang, F Glineur, J Louveaux, L Vandendorpe, Weighted sum rate maximization for downlink OFDMA with subcarrier-pair based opportunistic DF relaying. IEEE Trans. Signal Process. PP(99), 1-1 (2013)

24. W Dang, M Tao, H Mu, J Huang, Subcarrier-pair based resource allocation for cooperative multi-relay OFDM systems. IEEE Trans. Wireless Comm. 9(5), 1640-1649 (2010)

25. N Himayat, S Talwar, A Rao, R Soni, Interference management for $4 \mathrm{G}$ cellular standards [WIMAX/LTE UPDATE]. IEEE Comm. Mag. 48(8), 86-92 (2010)

26. Z Jin, L Vandendorpe, Resource allocation in multi-cellular DF relayed OFDMA systems, in IEEE Global Telecommunications Conference (IEEE, Houston, 2011), pp. 1-5

27. M Salem, A Adinoyi, H Yanikomeroglu, D Falconer, Opportunities and challenges in OFDMA-based cellular relay networksa radio resource management perspective. IEEE Trans. Veh. Tech. 59(5), 2496-2510 (2010)

28. M Salem, A Adinoyi, M Rahman, H Yanikomeroglu, D Falconer, Y-D Kim, E Kim, Y-C Cheong, An overview of radio resource management in relay-enhanced OFDMA-based networks. IEEE Commun. Surv. Tutorials. 12(3), 422-438 (2010)

29. DWK Ng, R Schober, Resource allocation and scheduling in multi-cell OFDMA systems with decode-and-forward relaying. IEEE Trans. Wireless Comm. 10(7), 2246-2258 (2011)

30. T Wang, L Vandendorpe, Iterative resource allocation for maximizing weighted sum min-rate in downlink cellular OFDMA systems. IEEE Trans. Signal Process. 59(1), 223-234 (2011)

31. M Chiang, CW Tan, DP Palomar, D O'Neill, D Julian, Power control by geometric programming. IEEE Trans. Wireless Comm. 6(7), 2640-2651 (2007)

32. M Chiang, Geometric programming for communication systems. Foundations Trends Commun. Inform. Theory. 2, 1-156 (2005)

33. BR Marks, GP Wright, A general inner approximation algorithm for nonconvex mathematical programs. Oper. Res. 26(4), 681-683 (1978)

34. SP Boyd, Software. http://stanford.edu/ boyd/software.html Accessed 1 Feb 2014

35. M Avriel, AC Williams, Complementary geometric programming. SIAM J. Appl. Math. 19(1), 125-141 (1970)

doi:10.1186/1687-1499-2014-71

Cite this article as: Jin et al:: Weighted sum of per cell min-rate maximization for multi-cell downlink OFDMA with opportunistic DF relaying. EURASIP Journal on Wireless Communications and Networking 2014 2014:71.

\section{Submit your manuscript to a SpringerOpen ${ }^{\circ}$ journal and benefit from:}

- Convenient online submission

- Rigorous peer review

- Immediate publication on acceptance

- Open access: articles freely available online

- High visibility within the field

- Retaining the copyright to your article

Submit your next manuscript at $>$ springeropen.com 